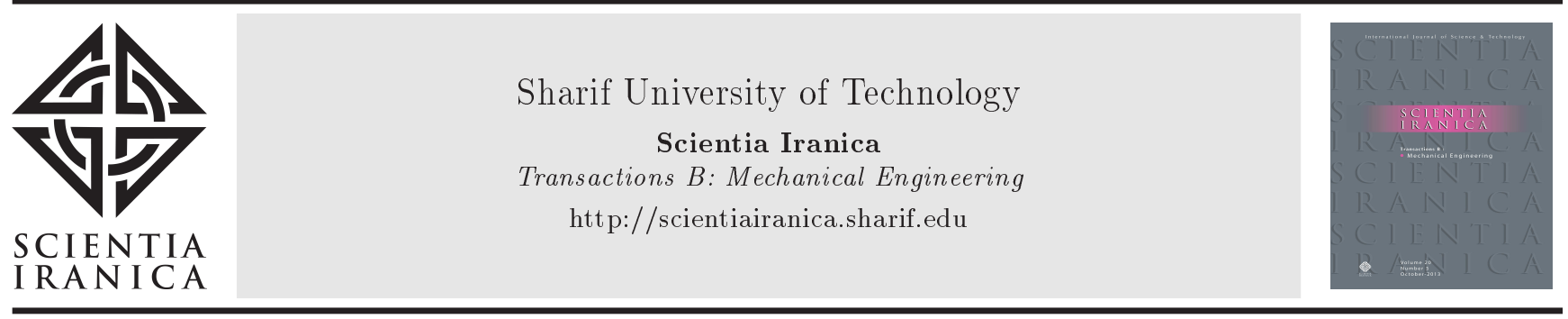

\title{
Parameter converting method for bifurcation analysis of nonlinear dynamical systems
}

\author{
H. Asghari and M. Dardel* \\ Department of Mechanical Engineering, Babol Noshirvani University of Technology, Babol, P.O. Box 484, Postal Code 47148-71167, \\ Mazandaran, Iran.
}

Received 3 April 2018; received in revised form 13 July 2018; accepted 22 October 2018

\author{
KEYWORDS \\ Parameter Converting \\ Method (PCM); \\ Sweeping parameter; \\ Bifurcation; \\ Poincaré map; \\ Continuation; \\ Nonlinear dynamics; \\ Time continuation \\ method; \\ Unlimited steady \\ solution method.
}

\begin{abstract}
For detecting the behavior of a dynamical system, bifurcation analysis is necessary to be conducted with respect to changes in the parameters of the system. In this study, based on the solution to ordinary differential equations from the initial value and parameters, a simple method is presented that can efficiently reveal different bifurcations of the system. In addition to its simplicity, this method does not require a deep physical and mathematical understanding of the problem and, because of its high precision and the speed of solutions, does not need to reduce the order of models for many complex problems or problems with high degrees of freedom. This method is called Parameter Converting Method (PCM), which has two steps. In the first step, the parameter varies as a function of time and, in the second step, time is expressed as the inverse of the assumed function. With this method at hand, bifurcation and amplitude-frequency diagrams and hidden attractors of some complex dynamics will be analyzed, and the sensitivity of the multi-potential well systems to initial conditions is studied. With this algorithm, a simple way to find the domain of high-energy orbit in bistable systems is obtained.
\end{abstract}

(C) 2020 Sharif University of Technology. All rights reserved.

\section{Introduction}

Many systems are described by dynamic equations. These systems exhibit overt and covert behaviors, and it is necessary to accurately analyze these systems. Investigation of the system's bifurcations for changes in the system parameters is the most important analysis. Because there is no analytical solution to complex dynamical equations, numerical solutions are used instead, or perhaps a semi-analytical solution is an appropriate choice in some cases; however, in many cases, a combination of these methods or the reduction

*. Corresponding author. Tel.: 01132334205

E-mail address: dardel@nit.ac.ir (M. Dardel)

doi: $10.24200 /$ sci.2018.50714.1832 of the order of equations through different methods can be effective. Due to the increasing development of computational systems and ease of accessibility, a combination of many numerical methods is proposed to investigate the dynamical system.

Perturbation techniques are the most known methods for analyzing nonlinear systems, although these methods are used for problems with simple geometry and low dimension. However, the major limitation of this method lies in the application of this method to problems with weak nonlinearity, which is usually introduced by a small parameter $\epsilon$. In this method, the solution of the problem is expressed in terms of power $\epsilon$; however, the computational cost of the system increases with an increase in the assumed terms and numbers of equations. There are different types of this method including multiple scales, averaging, Lindstedt- 
Poincare, straightforward expansion, variational iteration, etc. [1-3]. For solving this issue, the combination of numerical methods and perturbation techniques is a good strategy, as observed in the referenced study [4]; now, it is called Asymptotic Numerical Method [5,6]. This method is based on the path following, where each step of the solution is calculated on the basis of the solution of the truncated vector series. The application of vector Padé approximation leads to reducing steps of the solution and increasing the range of validity of the solution, where a new category of vectors causes the improvement of this method [7-9].

Algorithms based on the asymptotic numerical method, such as path-following technique, and nonlinear normal modes were developed to study the behavior of dynamic systems [10-12]. The basis of numerical solutions is continuation, in which the solution for a new parameter of the system is obtained through the previous solution. Different types of continuation methods are presented; pseudo-arclength continuation, parameter continuation, Moore-Penrose continuation, and piecewise linear continuation are more familiar instances [13-18]. Numerical methods including the periodic solutions, connecting orbits, discretization, and collocation are used for PDEs [19].

In a study conducted by Karkar et al. [20], the harmonic balance formulations for the continuation of periodic solutions were used. Karkar et al. [21] carried out a comparison between the harmonic balance method and the orthogonal collocation method for stiff nonlinear systems, which showed that the former outperformed the latter.

In many engineering problems, one may encounter equations with infinite or high degrees of freedom. To solve such problems, dimension reduction or reducedorder modeling is used, which is an active field in mathematics and engineering. These methods consist of center manifold method, Lyapunov-Schmidt (LS) method, Inertial Manifold (IM), various Galerkin methods, and Karhunen-Loeve or Proper Orthogonal Decomposition (POD) $[22,23]$. Based on POD, many methods have been presented like POD-Galerkin [24-28].

Generally, numerical methods are the essential parts of bifurcation analysis to observe how the system's behavior changes with the varying parameters of the system. There are many important and valuable research studies in this area, which are the main focuses of many researchers in different fields whose related algorithms are continuously improving [29-39]. On the basis of numerical continuation methods, AUTO, HOMCONT, MATCONT, Manlab, PyDSTool, COCO software, and other valuable software products are available. For example, Manlab is based on MATLAB programming and analyzes the stability and bifurcation at the equilibrium point. This software uses harmonic balance method and continuation algorithms to analyze stability and bifurcation and periodic orbits in the given dynamical system. The related algorithm is based on asymptotic numerical methods and Padé approximation [40]. In addition, Continuation Core and Toolboxes (COCO) project is written based on MATLAB and is a Toolbox for parameter continuation and bifurcation [41]. PyDSTool is a comprehensive software application for simulating physical systems (ODEs, DAEs, maps, and hybrid systems). PyDSTool is written in Python and, to increase its speed, its code is written in the language of FORTRAN and C [42].

AUTO is written in the Fortran language and is applied to the continuation and bifurcation problems in ordinary differential equations. Due to multi-core processing capabilities, this software is able to analyze the dynamic equations with high degrees and a high speed [43]. HomCont is a numerical toolbox for homoclinic bifurcation analysis. It is designed for use with AUTO. Specifically, HomCont deals with the continuation of codimension-one heteroclinic and homoclinic orbits to hyperbolic and saddle-node equilibria, including the detection of many codimension-two singularities and the continuation of these singularities in three or more parameters. MATCONT is a MATLABbased software product for the bifurcation study of dynamical systems. The aim of this toolbox is to provide an interactive environment for the continuation and normal form analysis of dynamical systems. This software can analyze the continuation of branch points in three parameters, the universal use of minimally extended systems, and the computation of normal form coefficients for bifurcations of limit cycles.

Classical attractors such as Lorenz [44], Rossler [45], Chua and Lin [46], Chen and Ueta [47], and other absorbers are excited through the unstable equilibriums (self-excited attractors). In terms of computation, in nonlinear dynamical systems, attractors in two forms of self-excited and hidden are presented. To achieve the self-excited attractor, it is possible to differentiate the path of oscillation in the nearby region of equilibrium point from the point on the unstable manifold with a transient solution. Hence, these types of absorbers are simply identified. In contrast, the achievement of hidden attractors is a difficult task, because they are not placed near the equilibrium point.

To find the self-excited attractor, conventional numerical methods can be used; however, to achieve the hidden attractor, it is necessary to propose a new solution method or extend available algorithms, since a transient solution similar to the self-excited attractor does not exist for the hidden attractor. In terms of applications, the issue of finding the hidden attractor is very important, since the knowledge about this hidden behavior can decrease the risk of a sudden and unexpected jump of the system [48]. To determine 
the location of the hidden attraction, many algorithms were developed and established [49-56].

Reducing the system's degrees of freedom without losing the main part of the behaviors of the system and reducing the required precision needs very strong mathematical tools. All semi-analytical solutions or reduced forms shift the bifurcation diagrams or, in some cases, may have a low degree of accuracy.

According to the above discussion, it is pleasure to provide a simple and efficient alternative method; a method that does not require a deep mathematical foundation and, instead, enjoys ease of implementation. In this work, a new method is presented based on the solution with ordinary differential equations. Due to its simplicity, it can easily be implemented and used to analyze the effect of changing parameters of the dynamic equation. This method does not require a deep understanding of mathematical concepts and, because of its high speed for solutions, does not need to reduce equations in many complex engineering problems, or problems with a high degree of freedom. Further, this algorithm can find the hidden attractor of the problem with a good degree of accuracy.

The method discussed here is based on a very slow change in parameters of the system during the solving process of Ordinary Differential Equations (ODE). This method can be simply implemented by available software products such as FORTRAN, C++, MATLAB, and other applications that have the ability to solve ODE equations. Moreover, this algorithm can be added to available continuation toolboxes such as MatCont, Hom Cont, Auto, etc.

In the first part of this work, the mathematical basis of this method is presented, and the implementation of this algorithm is outlined. In the second part, bifurcation diagrams of several complex dynamic systems are investigated and some of their hidden attractors are explored. In the third part, the amplitude-frequency diagram of the Duffing equation is investigated. A special case of the Duffing equation is treated, in which the equation has double-well potential and has hidden attractors under different initial conditions. In the final section, the conclusion along with some guidelines for future works is presented.

\section{Base of algorithm}

Many systems can be described by time-varying differential equations, as indicated below:

$$
\dot{x}=f[x(t), \alpha], \quad x \in R^{n}, \quad \alpha \in R^{n_{\alpha}},
$$

where $x$ is the state variable (a vector), $\dot{x}$ is the time derivative of the state variables, and $\alpha$ is the parameter of the system. Let's assume that the steady state response of the system for the assumed parameter set of $\alpha_{0}$ is known and the steady state response of the system for small variations in the parameter of the system, i.e., $\alpha=\alpha_{0}+\delta$, is desired, where $\delta$ is sufficiently small. This problem falls in the category of the continuation theory, in which a solution to the problem with varied parameters from the available solution is requested. There are some famous continuation algorithms, as shown in Figure 1, among which natural continuation and pseudo arc-length continuation are used to a greater degree.

Natural parameter continuation is similar to an iterative solver, by which the solution at one value of $\alpha$ is used as the initial guess for the solution at parameter $\alpha+\delta$, where $\delta$ is sufficiently small. Hence, with a small number of iterations, convergence to the true solution is applied. This algorithm is used in solvers such as LOCA. However, natural parameter continuation fails at turning points. The arclength continuation is based on the parameterization of the length of curve or arclength. This method is an approximation of the arclength in the direction towards the curve. The algorithm is a predictor-corrector method. The prediction step finds the point along the tangent vector; in addition, the corrector is usually Newton's method, or some variants, to solve the nonlinear system. This method can follow the curve through turning points and is used in solvers such as AUTO and Matcont.

The above-mentioned algorithms are mainly used in nonlinear algebraic equations, i.e., problems with in

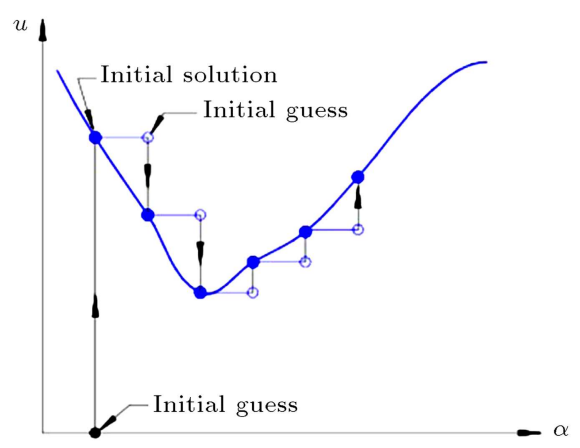

(a)

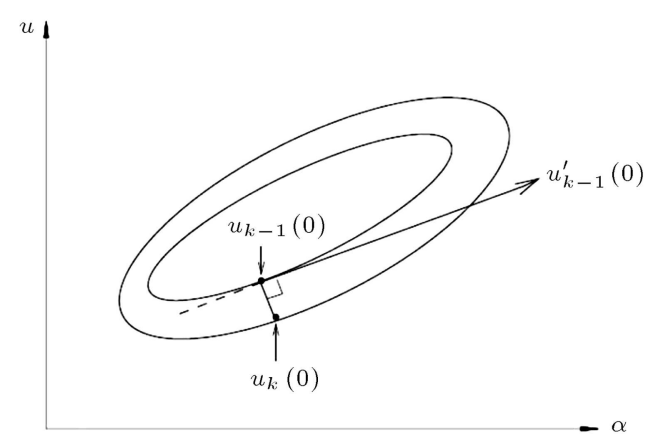

(b)

Figure 1. Continuation methods: (a) Natural and (b) arclength. 


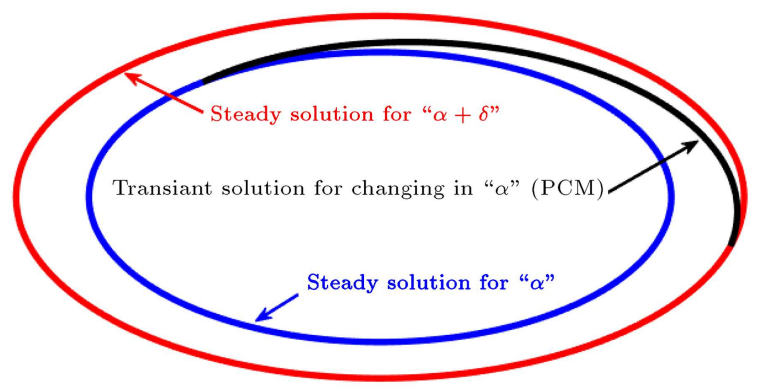

(a)

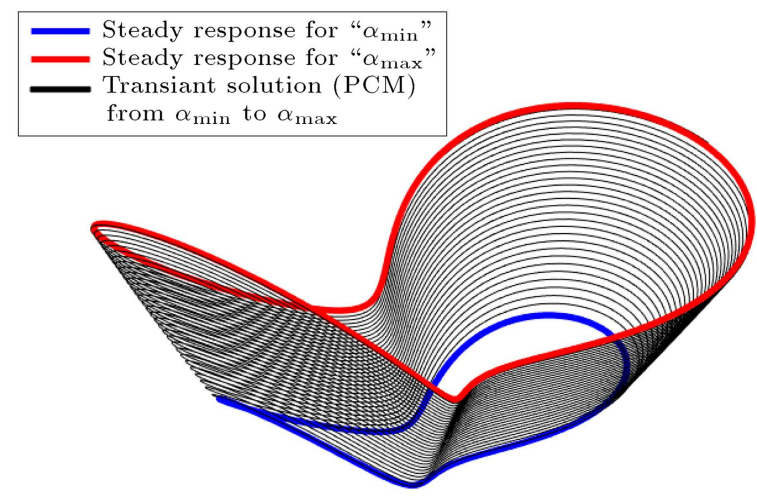

(b)

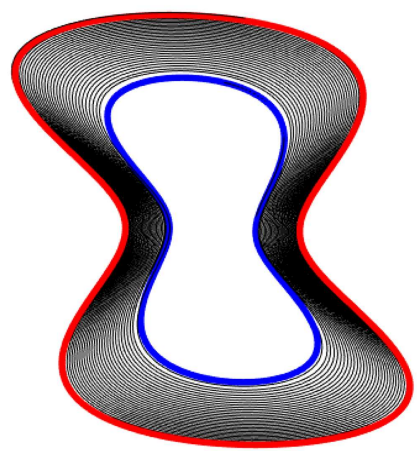

Figure 2. Schematic of how to apply the new method and its performance.

Eq. (1). The goal of the present work is to obtain the bifurcation of Eq. (1) with respect to the variation of parameter $\alpha$.

Let's assume that the steady state response of the system for the initial parameter $\alpha$ is available through solving Eq. (1). Now, if the variation of the system's behavior for a parameter of $\alpha$ in a region of $\alpha_{\min }<$ $a<\alpha_{\max }$ is desired, the following change of parameter (the first step of the parameter conversion) is applied:

Replacing "a" by:

$\begin{cases}\alpha_{\min }+\varepsilon\left(\alpha_{\max }-\alpha_{\min }\right), & \text { for increase (forward) " } \alpha " \\ \alpha_{\max }-\varepsilon\left(\alpha_{\max }-\alpha_{\min }\right), & \text { for decrease (backward) " } \alpha "(2)\end{cases}$

with,

$$
\frac{d \varepsilon}{d t}=\frac{1}{t_{2}-t_{1}} .
$$

Hence, $x_{n+1}=\varepsilon$ is the $n+1$ th state variable. The parameter $\alpha$ can be the amplitude of the exciting force, the frequency of the excitation force, or any other parameter of the system. Moreover, $t_{1}$ is the required simulation time to achieve the steady-state solution at parameter $\alpha_{\min }$ or $\alpha_{\max }$, i.e., the starting point, and $t_{2}$ is the virtual time (pseudo-time) and is similar to the parameter $s$ used in the arc-length continuation procedure. It should be noted that $t_{2}$ is not the real time; it only determines the rate of change in $\alpha$ or determines the parameter of $\varepsilon$. The greater $t_{2}$ is, the greater the resolution of solution for change from $\alpha_{\min }$ to $\alpha_{\max }$ will be.

In Figure 2, the schematic of this algorithm is shown. In Figure 2(a), the available steady state solution for parameter $\alpha$ (blue color) is shown, and the steady state solution for the varied parameter $\alpha+\delta$ (red color) is desired. The black line in this figure shows how the available solution for the present parameter of $\alpha$ asymptotically approaches the steady state solution for the varied parameter of $\alpha+\delta$. In Figure 2(b), in a typical problem such as Lorenz model, the sweeping of absorbers between the steady state solutions at $\alpha_{\text {min }}$ and $\alpha_{\max }$ is shown.

Basically, in this method, for a sufficiently long time, the dynamic equation of the system is analyzed for parameter of $\alpha_{\text {min }}$ and, accordingly, the steady state solution is obtained. This steady state solution is used as an initial solution for the parameter continuation of $\alpha=\alpha_{\min }+\delta$, similar to the natural continuation procedure described above. In the conventional natural continuation procedure, the equation of motion is solved again for the new parameter $\alpha=\alpha_{\min }+\delta$, and the steady state solution is obtained again. This is a time-consuming procedure and inefficient. However, another methodology is used in this paper. This methodology is similar to the natural continuation procedure, yet with some differences.

In the proposed algorithm, after obtaining the 
steady state solution for $\alpha_{\min }$, the time parameter is converted to $\alpha$ parameter (the second step of parameter conversion) as follows:

Conversion of time with parameter $\alpha$ :

$$
\left\{\begin{array}{r}
\alpha_{\min }+\left(\alpha_{\max }-\alpha_{\min }\right) \frac{T-t_{1}}{t_{2}-t_{1}}, \\
\text { for increase (forward) } \alpha \\
\alpha_{\max }-\left(\alpha_{\max }-\alpha_{\min }\right) \frac{T-t_{1}}{t_{2}-t_{1}}, \\
\text { for decrease (backward) } \alpha
\end{array}\right.
$$

where $t_{1}<T<t_{2}$. Now, if the equation of motion is solved again while the desired parameter of $\alpha$ varies according to Eq. (3) and, since the initial solution is in the steady state, the system will continuously remain in this state and the steady state solution will be obtained for variations in parameter $\alpha$ with a sufficiently high degree of accuracy. This algorithm can be implemented by software with an ODE solver.

Since the proposed algorithm is similar to the natural continuation method, it is necessary to give some additional description of this algorithm. The conventional natural continuation method is generally used in conjugation with nonlinear equations without time derivatives, in which through Taylor expansion of nonlinear equation around the previously obtained solution and linearization of equations or determination of the Jacobians of equations, the solution of the problem for a new set of parameters is obtained. Now, a similar procedure is proposed for time-dependent nonlinear equations, in which Runge-Kutta or method of solving ODEs is used here. By gradually varying the parameter of system and obtaining the local maxima for each of the states of the response, the bifurcation diagram of the system will be obtained. For this purpose, in MATLAB software, the findpeaks command can be used.

The main advantage of this algorithm is that while ODEs are solved, it is not required to solve the equations for a sufficiently long amount of time regardless of the transient response. In fact, in the previous methods, to check the bifurcation or the behavior of the system, the transient solution was first performed with discrete changes in parameters; then, the steady solution is evaluated. Of course, some algorithms are proposed to remove the transient solution. A simple algorithm commonly used to obtain the steady state response in this conventional method is shown in Figure 3(a).

Basically, in Parameter Converting Method (PCM), at an initially assumed parameter, the transient solution is obtained until the system reaches the steady state; after that, this steady state solution is then used to obtain the solution with a minor increase or decrease in the parameter of the system. In fact, with this strategy, although the continuous change of the desired parameter of the system is in process, the system is always in its steady state.

There is a critical point in ensuring the convergence of the solution in its steady state while the parameter of the system is continuously changing. This is for two reasons: The first reason is in the selection of step size that can be small as much as possible; for example, in the ODE solver of MATLAB, a step size up to $1.41 \times 10^{-14}$ can be selected (further, more stringent control parameters can be applied to the ODE solver); the second reason is that, in the case of the Runge-Kutta method of solving ODEs, the next value is determined by the present value plus a weighted average of four increments, where each increment is the product of the step size and an estimated slope is specified by the right-hand side of the differential equation. Hence, accordingly, it is similar to the Newton-Raphson method for solving nonlinear equations.

In other words, it can be noted that by removing the transient period of the solution, a significant increase in the speed of the solution is observed. This algorithm is shown in the flowchart of Figure $3(\mathrm{~b})$. The performance of the new algorithm is shown schematically in Figure 4, which shows high accuracy and resolution in a short amount of time in the evaluated diagram in the new algorithm, compared to the last procedure. For example, if one seeks to look into the behavior of the system through the conventional methods in four distinct parameters, in each of these parameters, after obtaining the steady response of the system, the exact behavior of the system is achieved regardless of whether the values of these parameters are near or far away from each other. Now, if one needs to examine the problem with the same runtime of the conventional method in the continuous investigation between the selected parameters, at less than $t_{2}^{*}$, a less accurate response in analyzing the behavior of the system is obtained, although the bifurcation diagram of the system with higher quality and continuous variation of the parameter is obtained. From another perspective, if the distinct and limited analysis of the parameters of the system is considered, the previous approach is more appropriate; however, if the continuous analysis of the parameter and a more complete analysis of the system's behavior in a shorter time period are considered, the new method is much more suitable.

In other words, in the case of using the conventional method, if the steady state of the system with some different parameters is required, the problem along with these parameters should be simulated and solved independent of each other. Each of these simulations requires a specific runtime; accordingly, the total runtime is the sum of them. However, for the present method, the problem is continuously solved in the specific region of the parameters, and the solution 


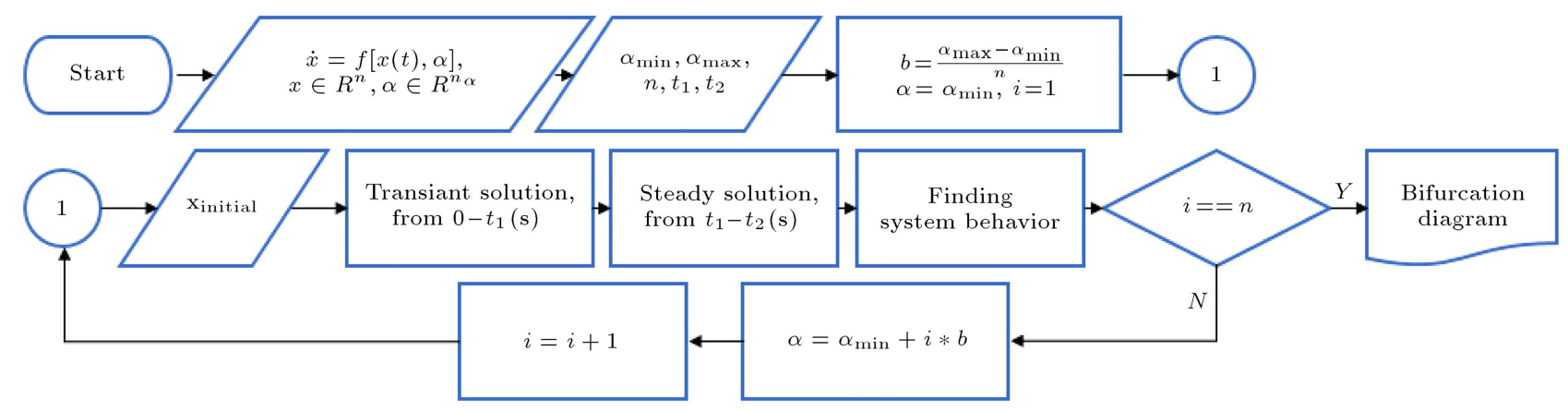

(a)

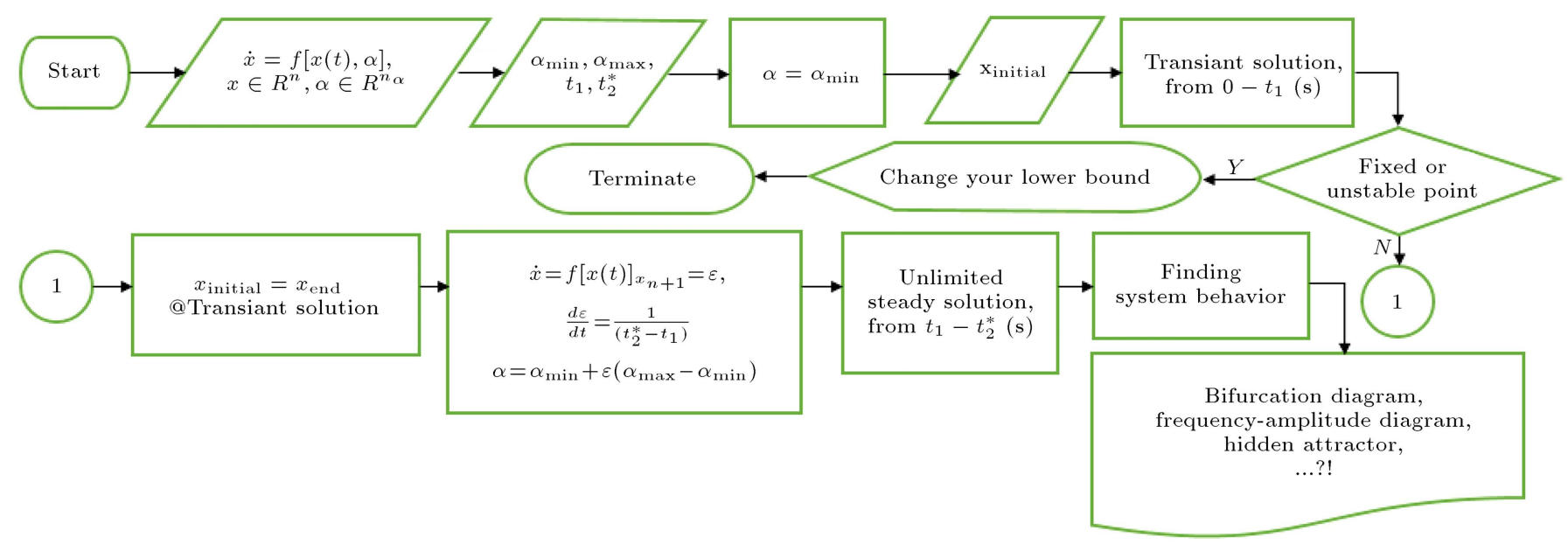

(b)

Figure 3. Methodologies to obtain the steady state solution: (a) Conventional and (b) the new proposed algorithm.

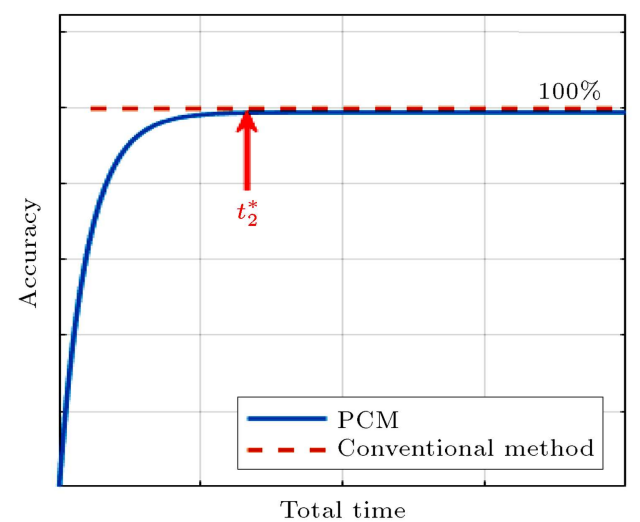

(a)

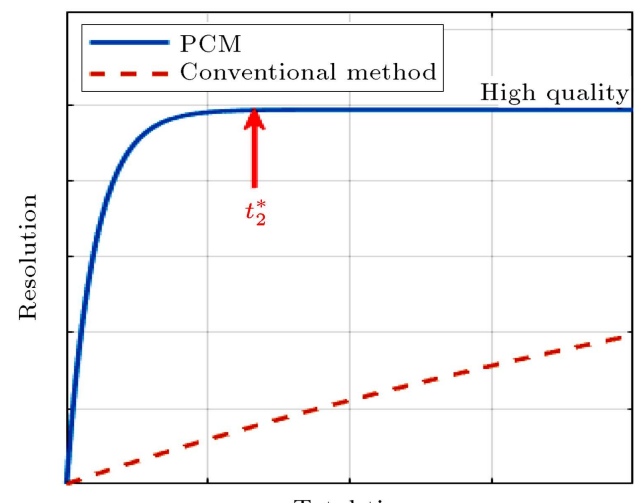

(b)

Figure 4. (a) Schematic of the solutions' accuracy in the new and conventional methods. (b) Schematic of the resolution diagram in two methods.

with all parameters is continuously obtained with high precision.

Figure 4(a) shows that the precision of the new method approach is higher than that of the conventional method; the conventional method gives the response of the system only with distinct parameters, while PCM gives the solution with all parameters. Figure 4(b) shows that the quality of the bifurcation diagram obtained by the new method is more than that of the conventional method. 


\section{Bifurcation diagrams and hidden attractors}

Now, the proposed algorithm is applied to different famous nonlinear systems to explore their different behaviors and show the effectiveness of the proposed algorithm.

\subsection{Chua's Circuit}

The circuit was first presented by Leon O. Chua in 1983; due to the complexity and simplicity of the equation and chaotic behavior of the circuit, it is known as "a paradigm for chaos" [46]. Equations of Chua's circuit are given as follows:

$$
\begin{aligned}
& \dot{x}=\alpha(y-x)-\alpha f(x) \\
& \dot{y}=x-y+z \\
& \dot{z}=-\beta y-\gamma z \\
& f(x)=m_{1} x+\frac{1}{2}\left(m_{0}-m_{1}\right)(|x+1|-|x-1|) .
\end{aligned}
$$

The behavior of this system with respect to the variation of parameter $\alpha$ in the range of $\left[\alpha_{\min }, \alpha_{\max }\right]=$ $[7,8.85]$ is examined here. The goal is to determine the behavior of the system and bifurcation diagrams and, also, find its hidden attractors. According to $\mathrm{Li}$ et al. [58], the following parameters and initial conditions are considered:

$$
\begin{aligned}
& \beta=12.0732, \gamma=0.0052, \\
& m_{0}=-0.1768, m_{1}=-1.1468, \\
& x(0)=-6.0468, y(0)=0.0839, z(0)=8.7739 .
\end{aligned}
$$

To determine the bifurcation diagrams, in this case, at first, a reduction in the control parameter is considered, and the response of system at $\alpha_{1}=8.85$ is determined. This response is shown in Figure 5 and, as is clear, the system has a complex behavior and chaotic behavior is exhibited even in a long simulation time. Generally, the first solution time must be long enough to ensure

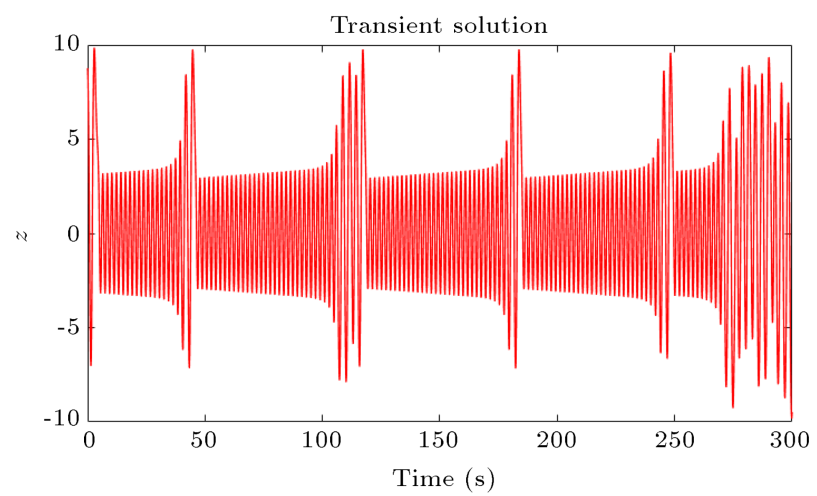

Figure 5. Transient solution in Chua's equation for $\alpha_{1}=8.85$. achieving a steady state response (if existed) with high accuracy.

Now, the intersection of a response with plane or section, desired state or final state of the solution is selected as an arbitrary condition or an initial value for the next solution, and the parameter $\alpha$ in comparison to the previous value changes into a small size $\delta$. Now, if Eq. (4) is solved again for this new value $\alpha=\alpha_{1}+\delta$, the steady-state response under new conditions will be obtained.

However, the noteworthy point to consider here is that it is not required to solve Eq. (4) for a long period of time since $\delta$ is sufficiently small to determine the steady state response for this new value; in addition, because the initial conditions are obtained from the previous steady solution, the steady response of the system is obtained for fractions of a second while it has a high degree of accuracy. Hence, with a continuous change in the control parameter, the solution is always in steady state. In a nutshell, due to solving ODEs by methods such as Rung-Kutta, an increase in the control parameter is synchronized with an increase in time.

Now, in accordance with Eq. (2), if the backward solution is used, the results shown in Figure 6 are obtained. This response is steady with respect to time, while it is transient with the control parameter, i.e., this response is the steady state summation of transient responses. In Figure 6(a), the backward solution of the differential equation with continuous variation of the control parameter in accordance with Eqs. (2) and (3) is shown with Cyan plot (transient solution). In accordance with the mentioned algorithm, if the peaks of this data are determined, as shown in the zoomed figure, the bifurcation diagram shown on the top part of this figure can be obtained. A similar procedure is applied, as shown in Figure 6(b), for forward solution. According to Figure 6 , the system shows chaotic behavior when exceeding $\alpha_{1}=8.85$, and it absorbs a strange attractor.

To prove the accuracy of the bifurcation diagram of Figure 6, the steady state response obtained with PCM and exact solutions are compared in Figure 7. For this purpose, in Figure 5, the response of the system at $T \in[7 E 4,7.01 E 4]$ is selected. At this pseudo-time, the control parameter is: $\alpha \in[8.41913,8.41975]$. If the average of the control parameter at this interval, i.e. $\alpha_{\text {ave }}=8.41944$, is selected and the exact solution is determined by solving the equations of motion, the comparisons of these solutions are shown in Figure 7. As seen, there is very good agreement between these results, showing the high accuracy of the proposed algorithm.

Hence, if the local maximums in the time response of Figure 5 in accordance with Eq. (3) and MATLAB findpeaks command are selected, the blue diagram in Figure 8 is obtained. Now, the effect of the solution 


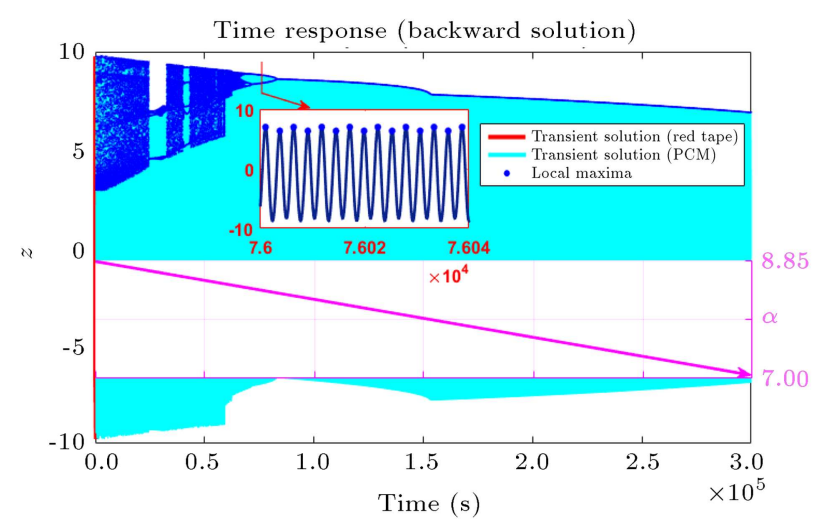

(a)

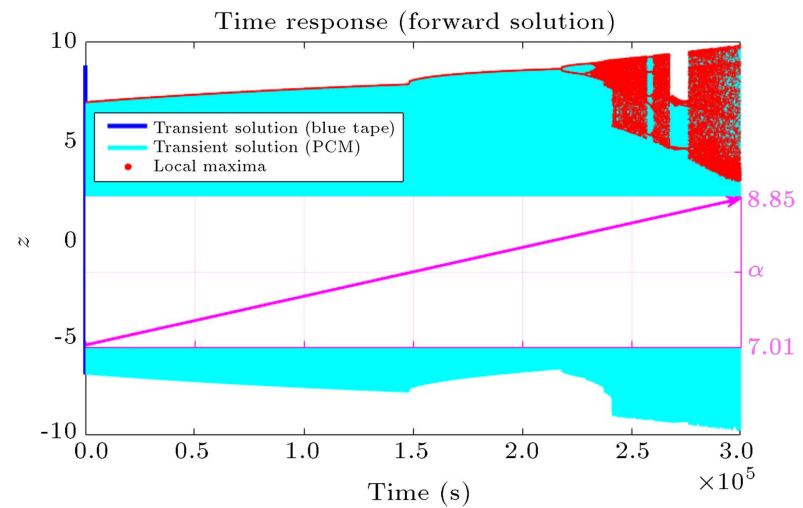

(b)

Figure 6. Transient solution with a new algorithm for changes in $\alpha$ from 8.85 to 7: (a) Backward solution and (b) forward solution.

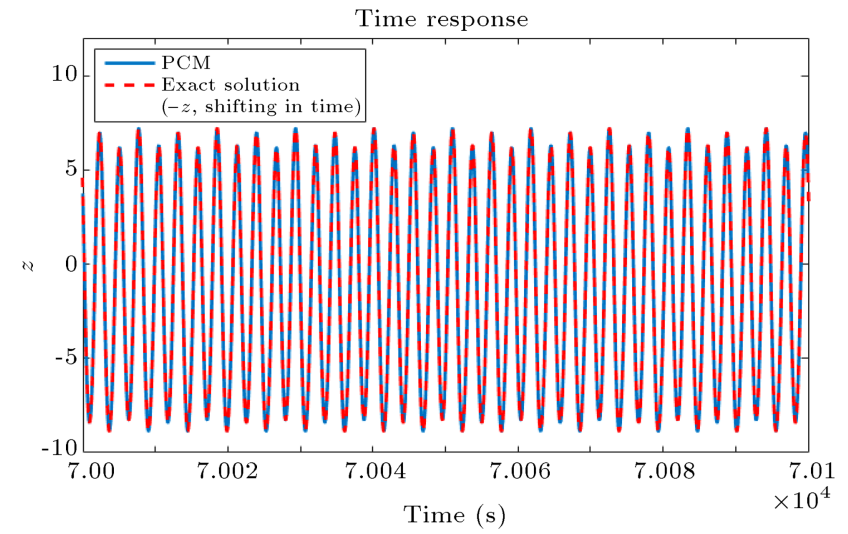

Figure 7. Comparison of the exact solution and transient solution minor changes $\alpha \in[8.41913,8.41975]$.

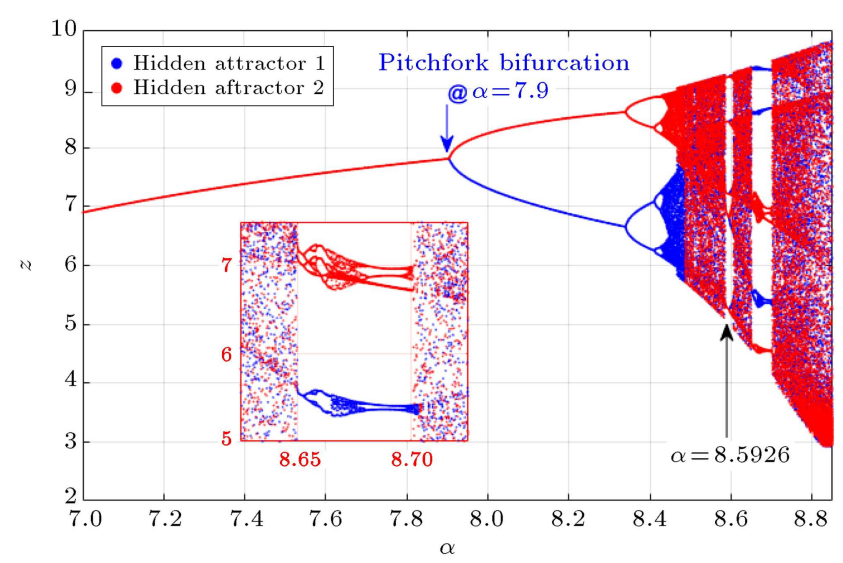

Figure 8. Bifurcation diagram of " $z$ " for changes in $\alpha \in[7,8.85]$; in the blue figure, the solution is $3 \times 10^{5}$ and $3 \times 10^{10}+10$ in the red figure.

time in this bifurcation diagram should be studied. If the pseudo-time varies from $3 \times 10^{5}$ to $3 \times 10^{10}+10$, the hidden behaviors of the system, including the hidden attractor, are obtained. These hidden attractors are revealed in the transition from the chaotic regions. With varying solution time, the pseudo-time step is reduced and, thus, we can exit the chaotic region with conditions different from those of the previous solution. Hence, the hidden attractors will be revealed.

These attractors are plotted in Figures 9 and 10. Of course, these attractors in [58] are obtained by mirroring the initial conditions (however, other initial conditions can also be used), while the hidden attractors are identified here with minor changes in the time required to solve the algorithm.

In Figure 10, different twin absorbers are shown. There are two absorbers that are symmetrical with respect to the origin [58].

In [58], Figure 9(b) has not been observed before; however, in PCM with minor changes in the total solution time of the system, hidden behaviors of the system in the vicinity of chaos can be found. In fact, this algorithm in the transition from chaos achieves the hidden behavior of the system. In the same study [58], the hidden attractor was found from this viewpoint such that, since Chua's Circuit was invariant under the transformation $(x, y, z) \rightarrow(-x,-y,-z)$, the two attractors must be symmetrical with each other with respect to the origin. In the conventional methods, different initial conditions in this hidden attractor can be achieved; however, in this method, without this property and with a slight change in the time of calculation or a change in the direction of the solution, forward or backward solution, these hidden attractors were obtained. It is important to note that for a simple system such as Chua, the properties of the system are accessible; however, in complex systems with higher degrees of freedom, there are more complex properties that may not be achievable. In PCM method, in transition from chaos, some of the properties of the system such as hidden attractors are achievable.

However, some questions still remain to be answered: Are these hidden attractors limited in number? How much is this minor change in solution time to identify all behaviors of the system? In this analysis, these hidden attractors are obtained with a minor 


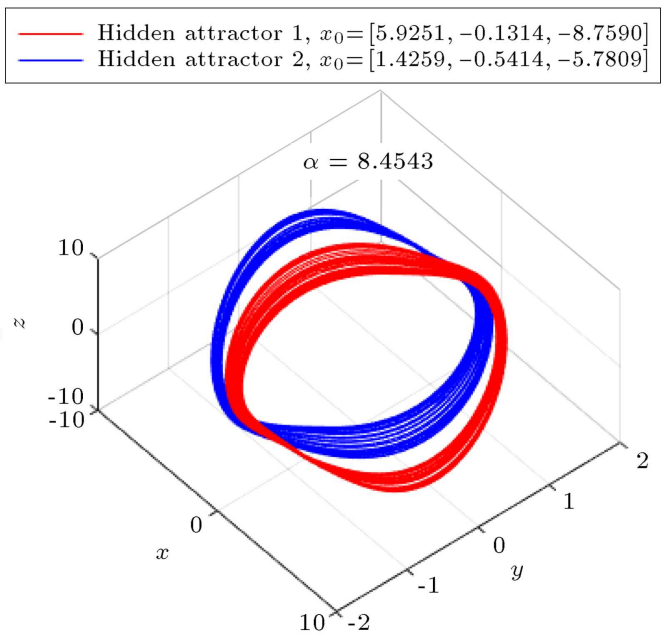

(a)

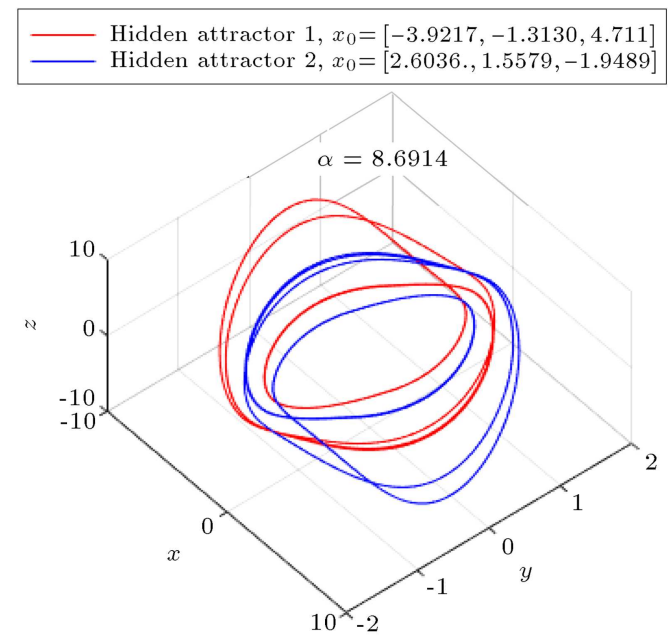

(b)

Figure 9. (a) A twin hidden attractor presented in [58]. (b) The new hidden attractor.

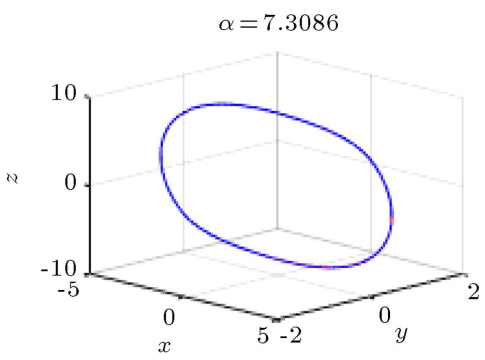

(a)

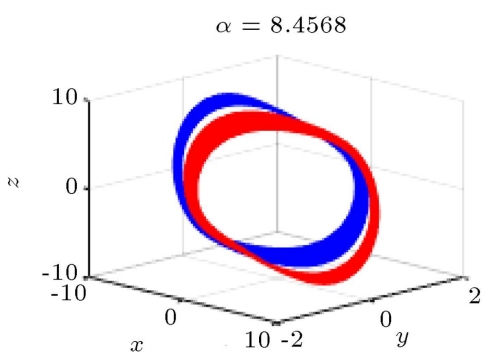

(d)

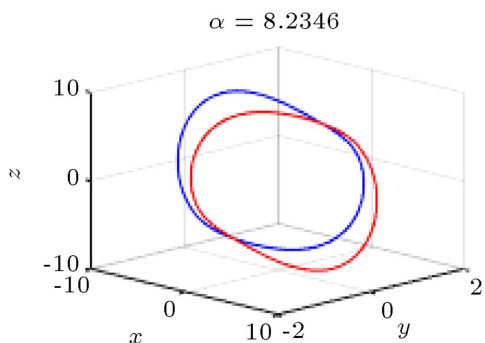

(b)

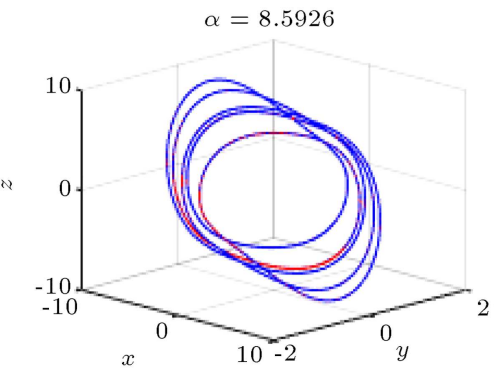

(e)

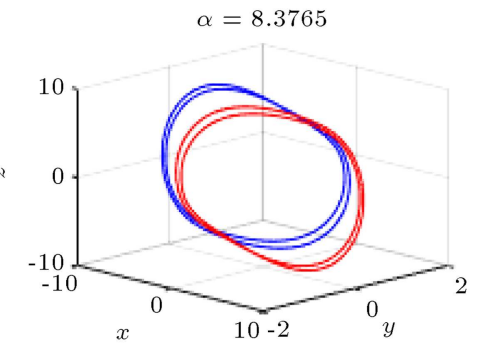

(c)

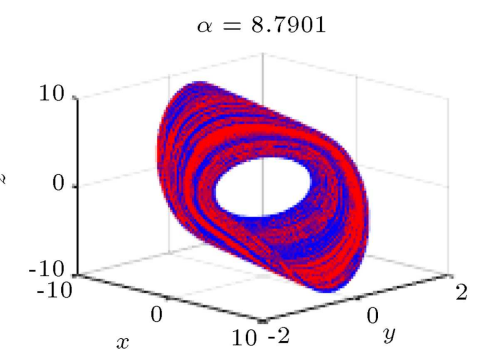

(f)

Figure 10. (a) Limit cycle with period 1. (b) Twin limit cycle with period 1. (c) Twin limit cycle with period 2, (d) Twin attractor. (e) Limit cycle with period 5. (f) Attractor.

change in the solution time; however, other parameters exist that may reveal other unknown behaviors of the system.

In accordance with Eq. (2), If the equation of motion is solved with an increase in the control parameter, there is a zero equilibrium point at $\alpha \leq 7$; however, with regard to Figure 2(b), the solution stops and, thus, it is necessary to change the starting point. The equilibrium point at $\alpha=7.01$ becomes unstable and, thus, a limit cycle occurs, which is a suitable solution to start and continue the algorithm.

Forward and backward solutions are shown in
Figure 11; however, which of them is more accurate? In accordance with the exact solution and Figure 12, the solution obtained with backward solution is more accurate; however, both solutions have an acceptable degree of accuracy.

Generally, if backward sequence is used instead of forward sequence, the obtained solution has a higher degree of accuracy, except the chaos. In Figure 13, although the simulation time for Chua's equations is reduced, the proposed algorithm enjoys good accuracy.

Bifurcation points in Figures 8 and 13 in $\alpha=7.9$ are a pitchfork bifurcation and period doubling in 


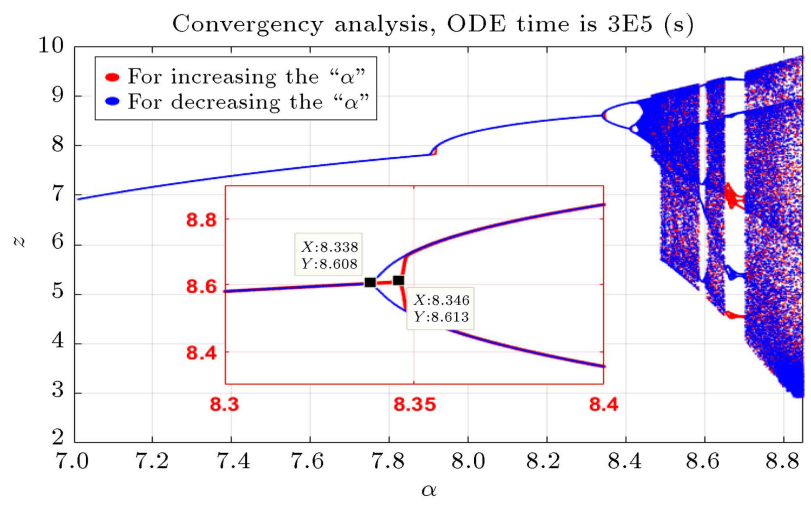

Figure 11. Comparison of increasing and decreasing solutions in Chua's equation.

$\alpha=8.34 ;$ in other words, there are two absorbers in two different regions, and each of these absorbers is presented at $\alpha=8.34$. For example, in Figure 10(b), in $\alpha=8.234$, two attractors have period one and, accordingly, a pitchfork bifurcation occurs at $\alpha=7.9$.

The effect of simulation time on the convergence analysis of the first period 2 is examined in Figure 14. As observed earlier, with an increase in the simulation time, the accuracy of predicting this behavior increases and high accuracy is achieved even in the short time analysis. Moreover, the backward solution converges more rapidly.

In the numerical solution of ordinary differential equations, methods such as Euler, Taylor series, linear multistep, and Runge-Kutta's are used. In all of these methods, the parameter of time step is used to integrate the differential equation in the considered domain through several steps. In these algorithms, to increase the accuracy and reduce the variation of solution, adaptive step-size selection techniques are used, meaning that if the time step becomes smaller, the accuracy of the solver increases, and vice versa [59].

Among the existing software applications, MAT-

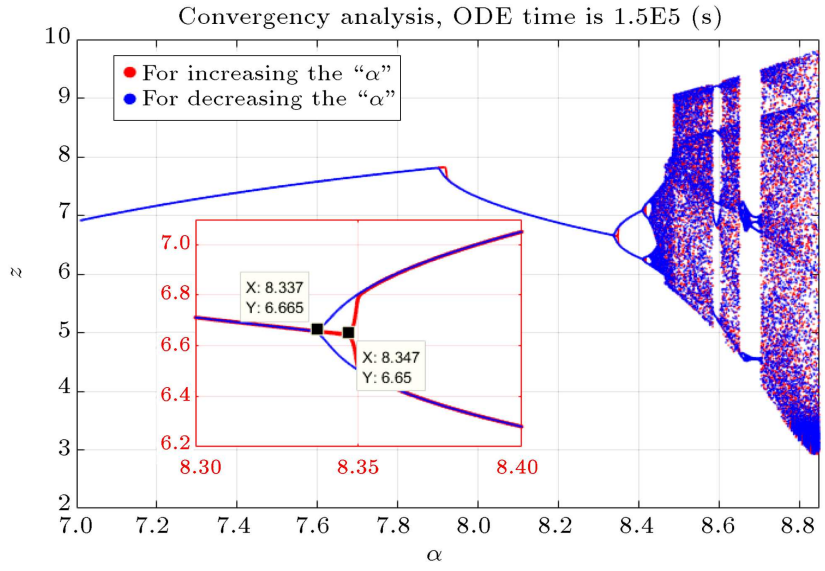

(a)

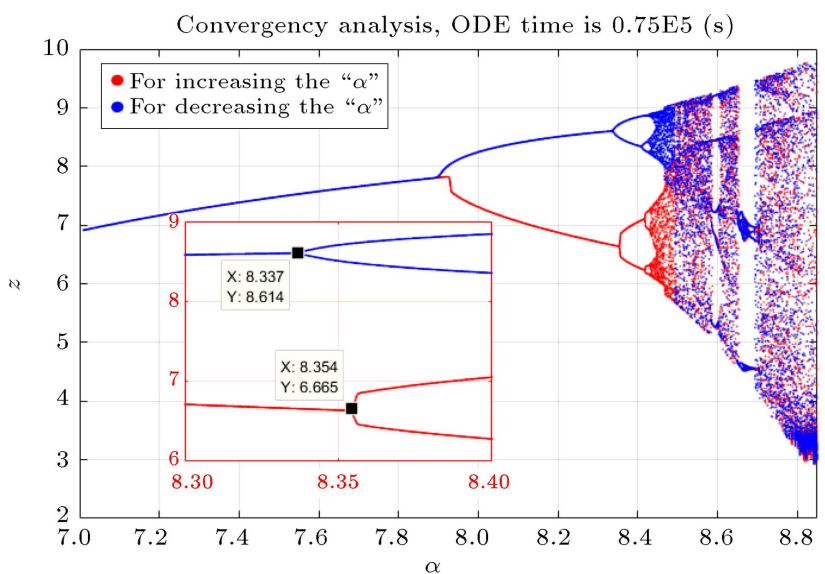

(b)

Figure 13. Investigating the effect of simulation time on the convergence of solution in forward and backward analysis.

LAB is an appropriate choice due to its convenience of interface. Using the ode 45 command in MATLAB is a simple way to solve ordinary differential equations. This command is based on Explicit Runge-Kutta $(4,5)$

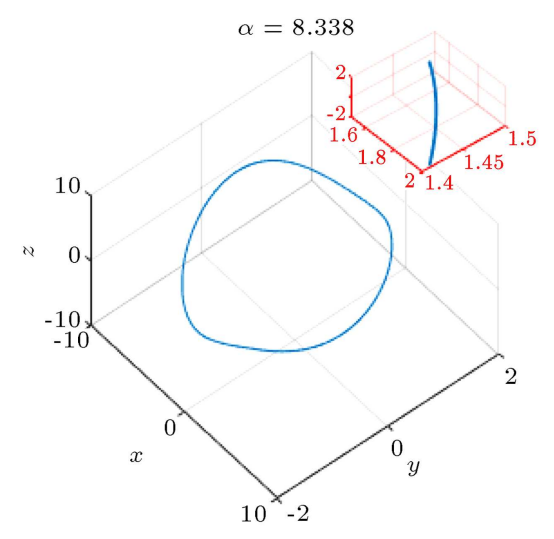

(a)

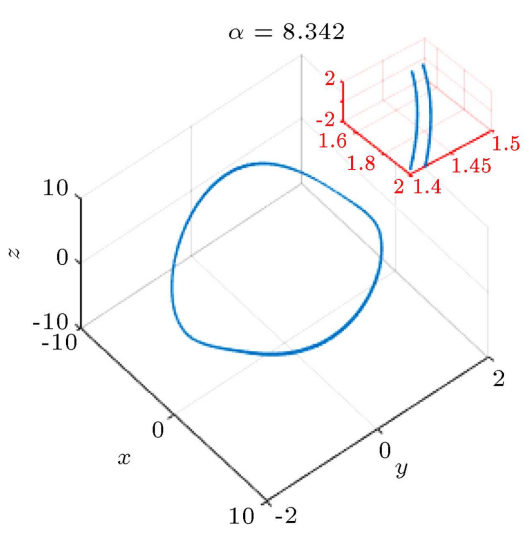

(b)

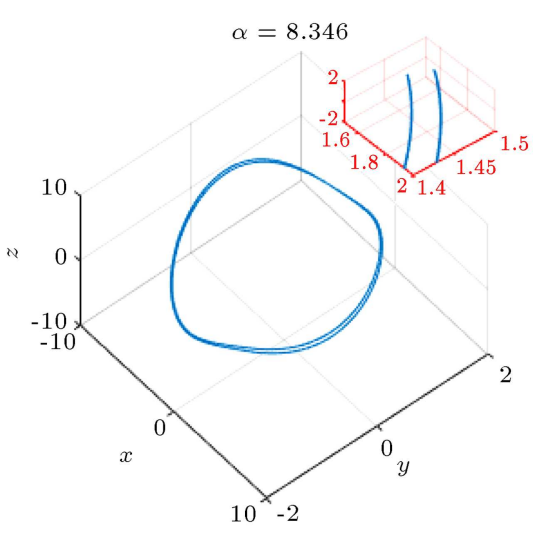

(c)

Figure 12. Comparison of the accuracy of forward and backward solutions at the first point bifurcation point with period 2 . 


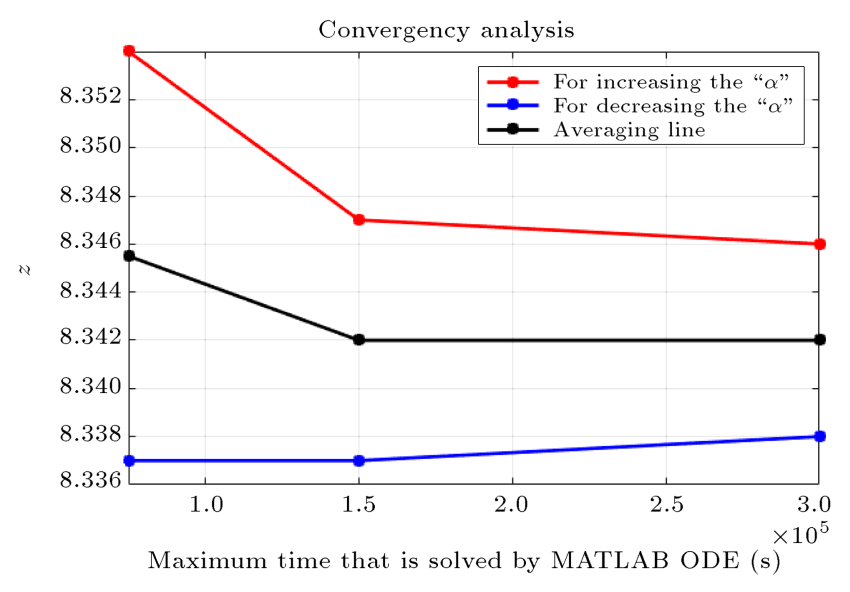

Figure 14. Comparison of the effect of an increase or a decrease in simulation time on the prediction point of the first period 2 behavior.

formula; in this method, the next step of the solution only requires one previous step. Generally, in most cases, ode 45 is the best choice for the first analysis and solution [60].

\subsection{Lorenz system}

Ed Lorenz from MIT University in 1963 tried to improve weather forecasting and discovered the first chaotic attractor. Lorenz [44] reduced the NavierStokes equations for convective L'enard fluid flow into the three first orders of coupled nonlinear differential equations and demonstrated the idea of sensitive dependence upon initial conditions and chaos. The Lorenz equations are as follows:

$$
\begin{aligned}
& \dot{x}=p(y-x), \\
& \dot{y}=x(r-z)-y, \\
& \dot{z}=x y-b z,
\end{aligned}
$$

where $x$ is the rate of convective overturning, $y$ is the horizontal temperature overturning, and $z$ is the vertical temperature overturning; the parameter $p=P$ is proportional to the Prandtl number, $r=R$ is proportional to the Rayleigh number, and $b=K$ is a number proportional to the physical proportions of the region under consideration [57].

In Figures 15 and 16, the bifurcation diagram of the Lorenz system for the $50<r<250$ is shown. At first, the forward solution with an initial parameter of $r=50$ and the initial condition of $[x, y, z]=[0,1,0]$ until $t_{1}=50$ is obtained. This time is selected such that the transient solution is diminished. Now, by applying the PCM and solving again the equations of motion until $t_{2}=6 \times 10^{5}$ and using forward solutions, the complete bifurcation diagrams of the system are obtained.

The bifurcation diagram shows that the system

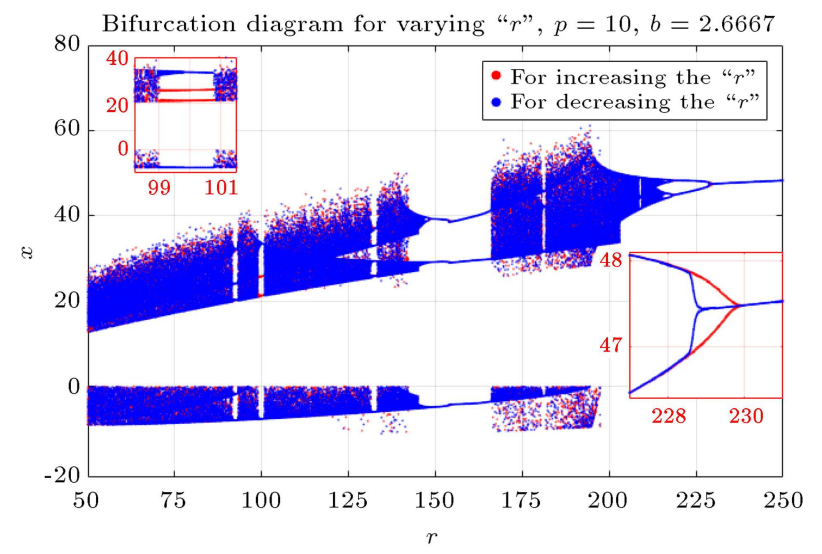

Figure 15. Bifurcation diagram for the state of $x$ in the Lorenz system at $50<r<250$.

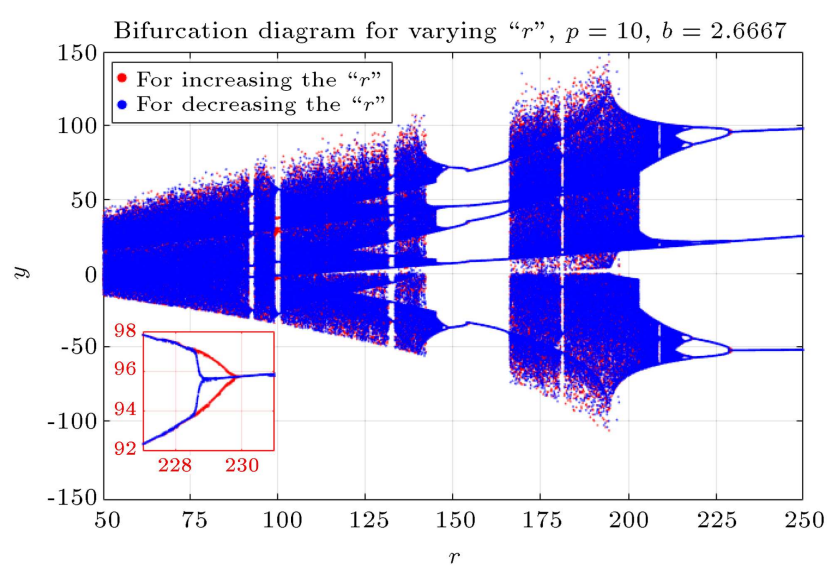

Figure 16. Bifurcation diagram for the state of $y$ in the Lorenz system at $50<r<250$.

shows chaotic behavior in $50<r<91.5$, period 5 for $x$, and period 7 for $y$ in $r=92$, periods 2 and 6 for $x$ and $y$ in $218.4<r<229.7$, and finally periods 1 and 3 for $x$ and $y$ in $229.7<r<250$. Figure 17 validates the bifurcation diagram around the bifurcation point, and shows that forward solution has a higher degree of accuracy. Moreover, some of the periodic solutions shown in bifurcation diagrams of Figure 15 are given in Figure 18, which are obtained from direct solutions of the Lorenz equations such as $r=100.3$.

The bifurcation diagram showed that at $r=100$ and in transition toward chaos, two modes of response can be observed. If the solution time of backward solution is different from forward solution, then several twin attractors can be obtained, some of which are shown in Figure 19.

The twin attractor in the Lorenz system can be justified, because this system has the following properties:

i. Symmetry $(x, y, z) \rightarrow(-x,-y,-z)$ for all values of the parameters; 


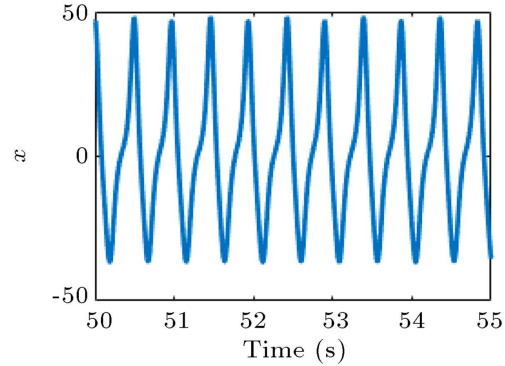

(a)

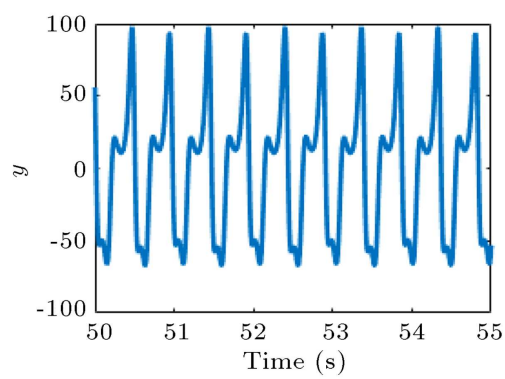

(d)

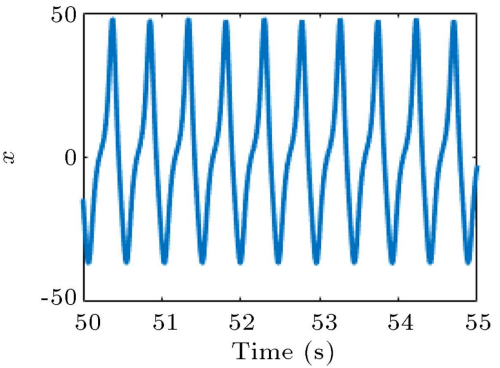

(b)

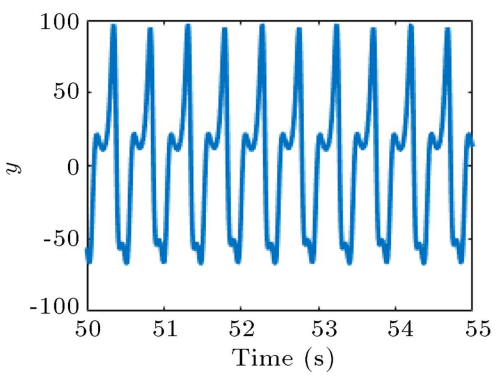

(e)

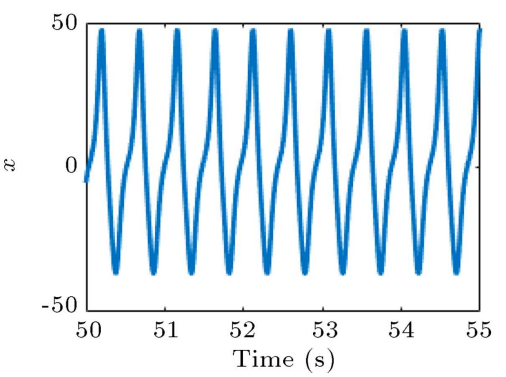

(c)

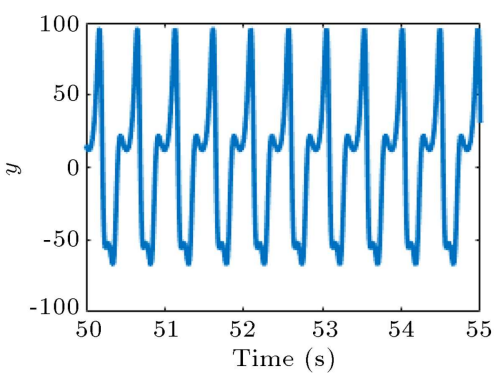

(f)

Figure 17. Validation of the bifurcation diagram with direct simulation of the Lorenz equation in the zoomed region shown in the bifurcation diagram: (a) $r=228$, period 2, (b) $r=229$, period 2, (c) $r=230.5$, period 1, (d) $r=228$, period 6 , (e) $r=229$, period 6 , and (f) $r=230.5$, period 3 .

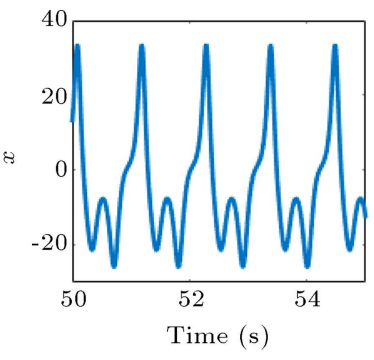

(a)

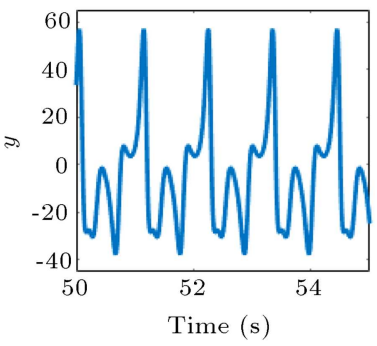

(e)

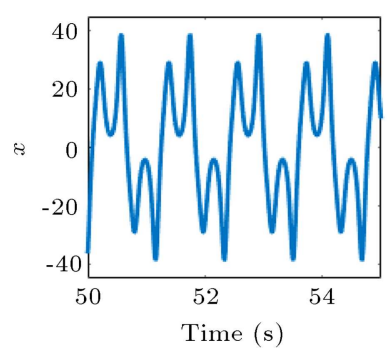

(b)

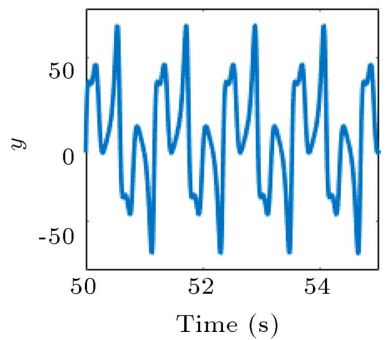

(f)

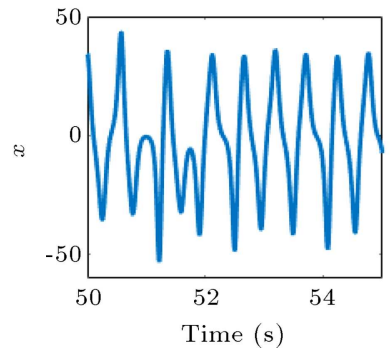

(c)

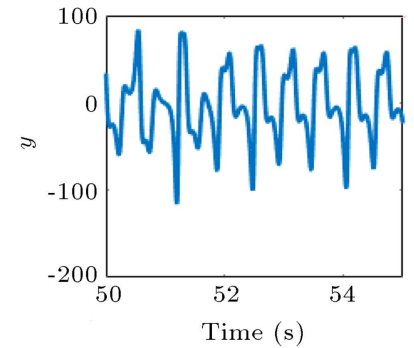

(g)

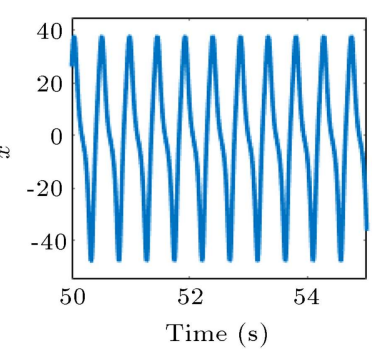

(d)

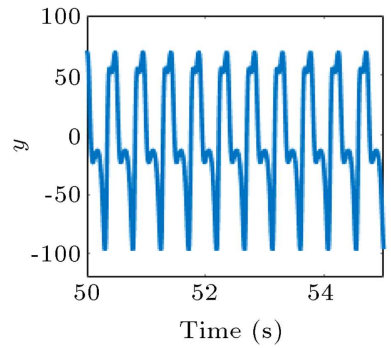

(h)

Figure 18. Validation of the bifurcation diagram with direct simulation of the Lorenz equation in some specific point: (a) $r=100.3$, period 2, (b) $r=155$, period 3, (c) $r=193$, chaos, (d) $r=240$, period 1, (e) $r=100.3$, period 4 (f) $r=155$, period $5,(\mathrm{~g}) r=193$, chaos, and (h) $r=240$, period 3 .

ii. The $z$-axis $(x=y=0)$ is invariant (i.e., all trajectories that start on it also end on it) [57].

As a result, in accordance with the first property with changes in initial conditions of the solution, all twin attractors can be found. However, this algorithm achieved the hidden attractors without the use of this property. This means that if the system contains an unknown behavior, this algorithm can reveal them.

\subsection{Rossler model}

The system is credited to O. Rossler and is used for modeling chemical kinetics [57,61]. 


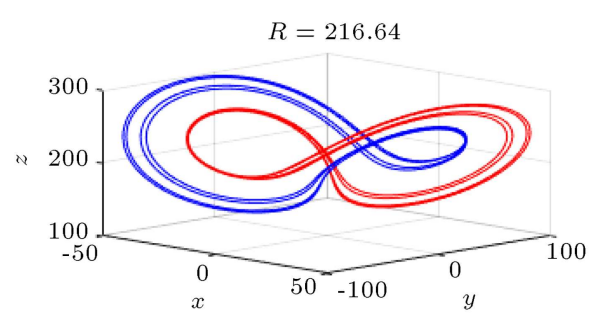

(a)

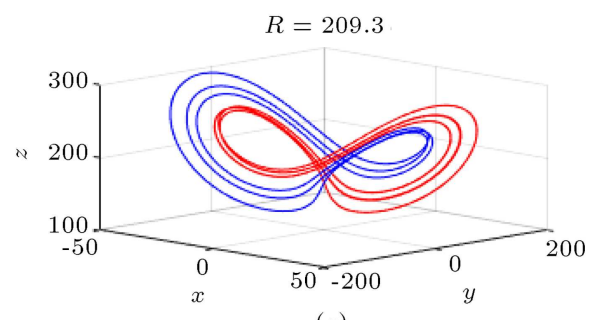

(c)

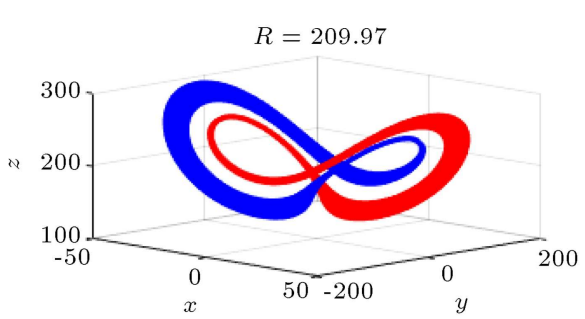

(b)

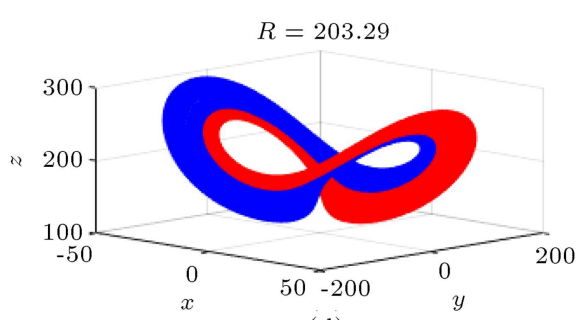

(d)

Figure 19. Several twin hidden attractors in the Lorenz system.

$$
\begin{aligned}
& \dot{x}=-(y+z), \\
& \dot{y}=x+a y, \\
& \dot{z}=b+(x-c) z,
\end{aligned}
$$

where $x, y$, and $z$ are the dynamic variables, and $a$, $b$, and $c$ are the system parameters. In Figure 20, the bifurcation diagram of Rossler model for $0.2<a<$ 0.55 is shown. For this purpose, at first, the governing equations of the system for $a=0.2$ and with the initial conditions $[x, y, z]=[0,0,0]$ until $t_{1}=300 \mathrm{~s}$ are solved such that the steady state solution is achieved.

Now, in accordance with PCM, if the governing equations are solved through Eq. (2) from the end state of the available solution until $t_{2}=3 \times 10^{5} \mathrm{sec}$, such that $a$ linearly changes from 0.2 to 0.55 , then the bifurcation diagram of Figure 20 with good accuracy is achieved. If $a=0.55$ is selected, the diagram can be obtained recursively. By selecting $a=0.55$, this figure can be obtained at backward solution.

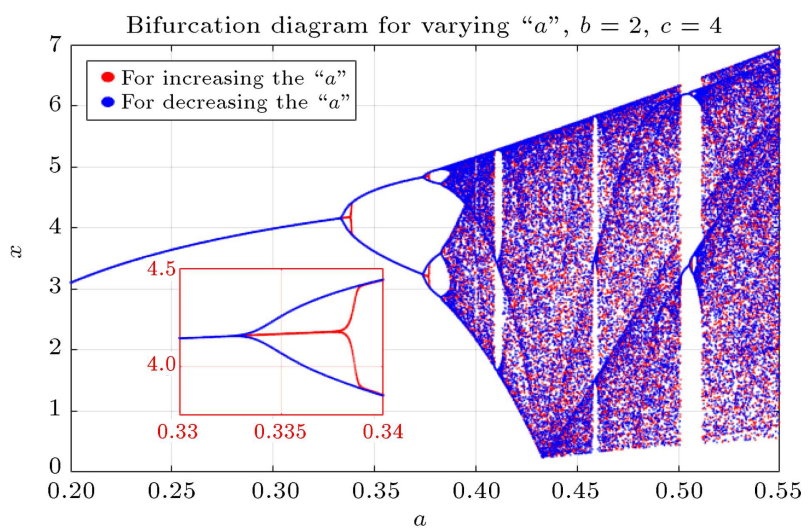

Figure 20. The bifurcation diagram for Rossler model for $0.2<a<0.55$.
This bifurcation diagram shows that the equation shows period one behavior in $0.2 \leq a \leq 0.333$ and period two behavior in $0.333 \leq a \leq 0.374$. With the increasing parameter, period doubling continues and chaotic behavior is created. Then, the periodic windows disappear, and chaotic behavior is diminished and the behavior with three periods at $a=0.41$ is revealed. Finally, the chaotic behavior is shown again.

In Figure 21, the accuracy of PCM is evaluated through the comparison of the values shown in the bifurcation diagram and the results obtained with a direct solution to the governing equation of motion.

Figure 22 shows the validation of the bifurcation diagram for other values of the control parameter, which indicates very good adaptability and high accuracy of this algorithm at sensitive locations such as $a=0.41,0.4582$, and 0.5031 .

In Figure 23, the bifurcation diagram of Rossler model for $0.1<b<2$ is shown. For this purpose, at first, the governing equations of the system for $b=0.1$ with the initial conditions $[x, y, z]=[0,0,0]$ until $t_{1}=$ $300 \mathrm{~s}$ are solved to achieve the steady state solution. Hence, by considering the steady state of this solution, applying the mentioned algorithm, solving the equation of motion until $t_{2}=3 \times 10^{5}$, and varying $b$ linearly from 0.1 to 2 , then Figure 23 with good accuracy is achieved. For backward solution, this procedure is applied from $b=2$.

The resulting bifurcation diagram shows that, for backward solution, the system has periodic one behavior in $1.44 \leq b \leq 2$, period two behavior in $0.857 \leq b \leq 1.44$, period doubling, periodic windows, chaotic behavior, and chaos disappearance.

In Figure 24, the bifurcation diagram of Rossler model for $1<c<45$ is shown. For determining this diagram, at first, the governing equation of motion for 


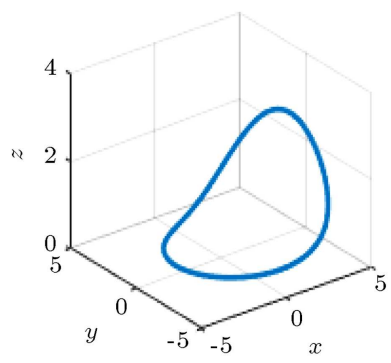

(a)

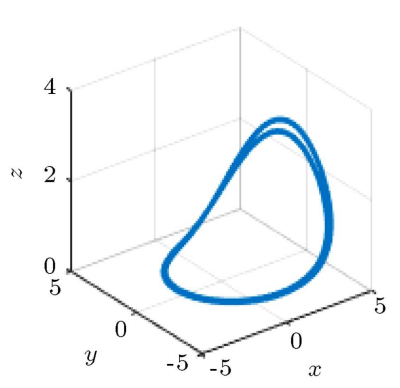

(b)

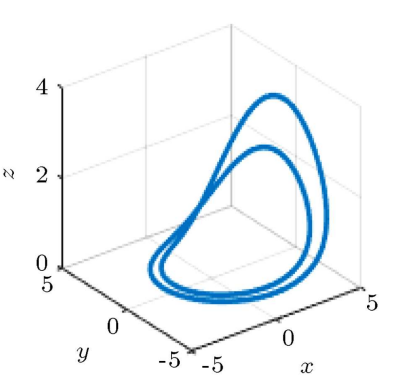

(c)

Figure 21. Validation of the bifurcation diagram for different values of the control parameter of Rossler model (in the zoom range): (a) $a=0.33$, period 1 , (b) $a=0.335$, period 2, and (c) $a=0.34$, period 2 .

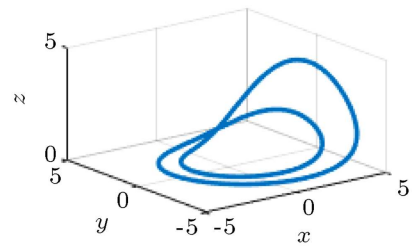

(a)

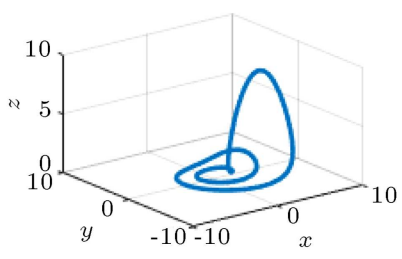

(d)

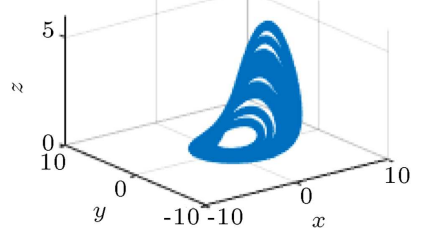

(b)

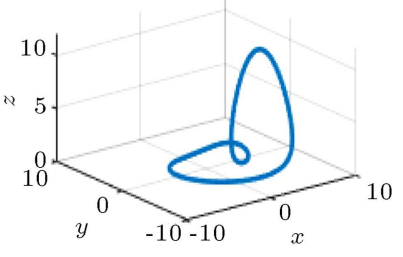

(e)

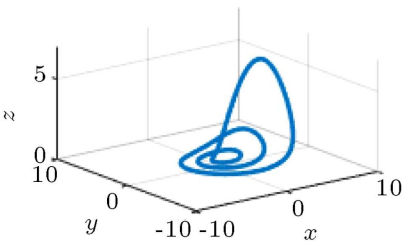

(c)

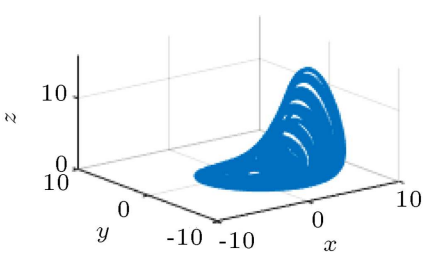

(f)

Figure 22. Validation of the bifurcation diagram at different values of the control parameter of Rossler model: (a) $a=0.3547$, period 2, (b) $a=0.398$, chaos, (c) $a=0.41$, period 3, (d) $a=0.4582$, period 3 , (e) $a=0.5031$, period 2, and (f) $a=0.55$, chaos.

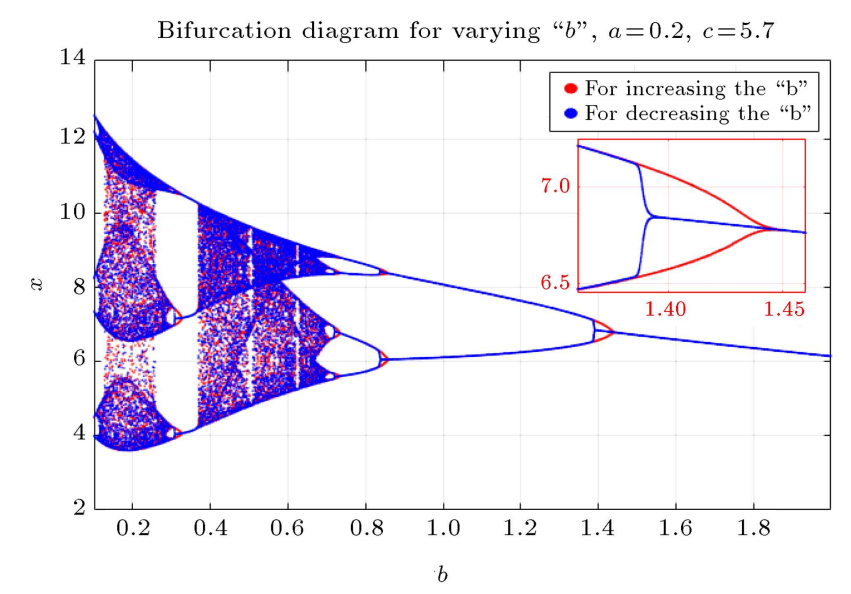

Figure 23. Bifurcation diagram for variation of the control parameter in Rossler model for $0.1<b<2$.

with initial conditions $[x, y, z]=[0,0,0]$ until $t_{1}=300 \mathrm{~s}$ is solved such that the transient response is diminished and the steady state response is achieved. Now, in accordance with the presented algorithm, from the end response of the previous solution at $c=1$, if this

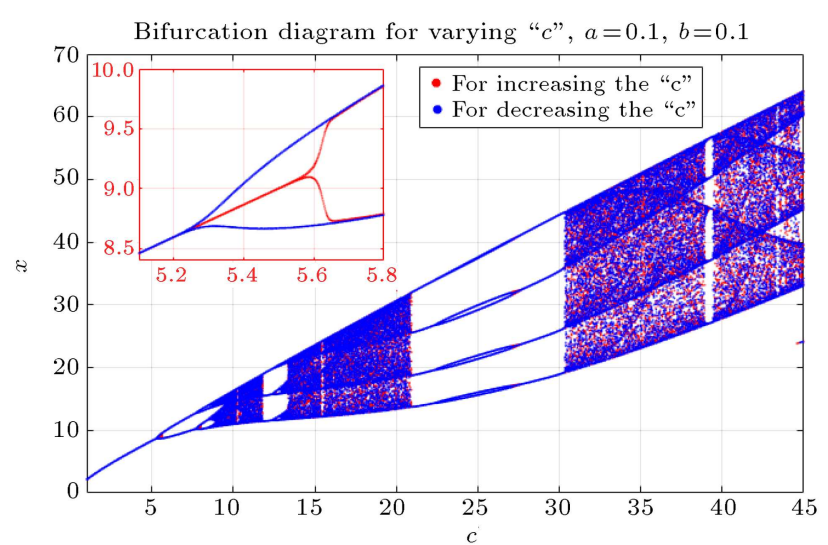

Figure 24. Bifurcation diagram for Rossler model for variation of the control parameter of $c$ in the domain of $1<c<45$.

control parameter linearly changes until $t_{2}=3 \times 10^{5}$, the bifurcation diagram can be determined. For the backward solution, $c=45$ is selected.

It should be mentioned that by increasing the control parameter, if the period of response varies 


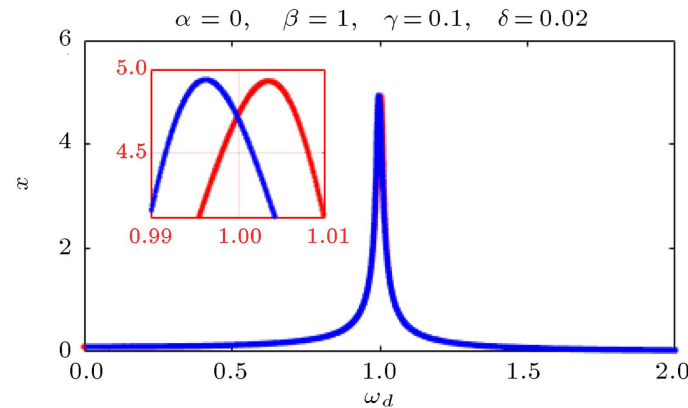

(a)

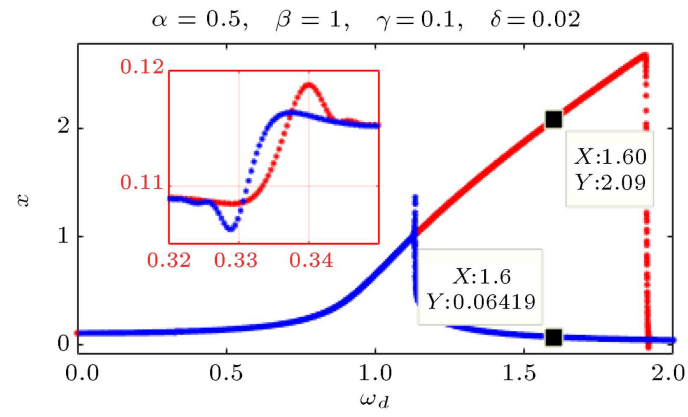

(c)

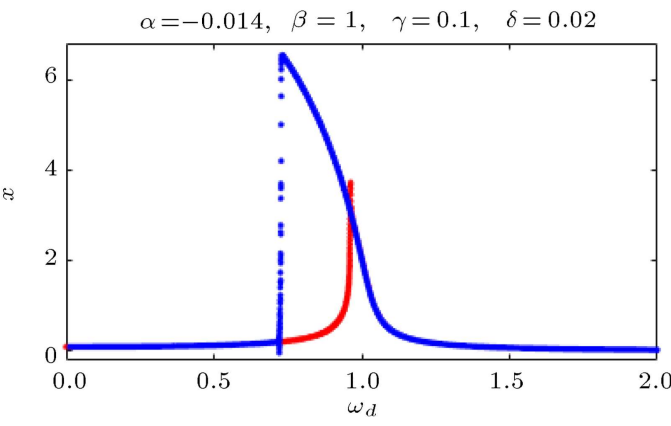

(b)

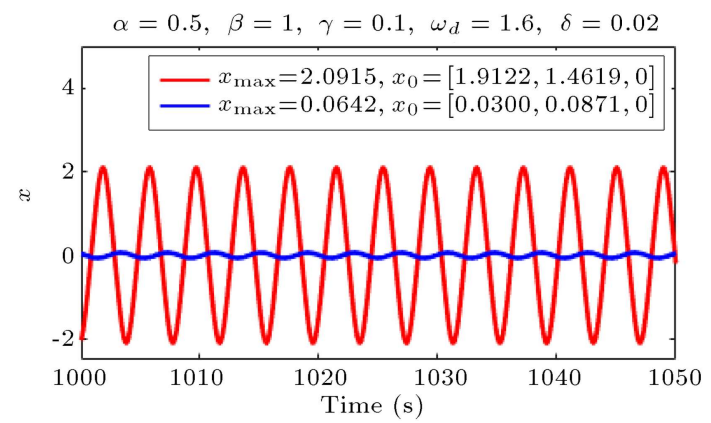

(d)

Figure 25. Frequency response curve of the Duffing equation in forward and backward solution of exciting frequency for different values of the system and exciting parameters: (a), (b) and (c) frequency responses of the Duffing equation, and (d) the validation of the solution.

from one to two, the backward solution becomes very close to the accurate solution (even is agreement with it), and vice versa. As a result, if the system is examined in a short amount of time after determining the overall behavior of the system, for increasing the accuracy of solution, forward or backward solutions can be used. However, if the system undergoes a sequence of two-to-one and one-to-two bifurcations, it is better to discretize the solution interval. For example, in Figure 23, for $0.1<b<0.2$, the backward solution is of good accuracy; for $0.2<b<2$, the forward solution has good accuracy; in addition to these, in Figure 24, for $1<c<25$ and $25<c<45$, the backward solution and forward solutions have good accuracy, respectively. However, finding these intervals can be obtained by solving the equations in a short amount of time, and it is not necessary to accurately determine them. Even if we do not use this method, generally, forward or backward solution has enough accuracy.

\subsection{Frequency-amplitude diagram: Duffing oscillator}

Now, the frequency-amplitude curve of the Duffing oscillator [62] is investigated by the presented algorithm. The Duffing oscillator is one of the most known models for studying different nonlinear behaviors of structural systems. The equation of this oscillator is as follows:

$$
\ddot{x}+\delta \dot{x}+\beta x+\alpha x^{3}=\gamma \cos \left(\omega_{d} t\right) .
$$

In Eq. (7), $x$ and $\dot{x}$ are the dynamical variables; $\delta, \beta$, $\alpha, \gamma$, and $\omega_{d}$ are the parameters of the system; $\gamma$ and $\omega_{d}$ are the amplitude and frequency of the excitation force. This oscillator for $\beta>0$ has non-linear spring force; for $\alpha>0$, the spring is called hardening and that for $\alpha<0$ is softening [63].

To analyze the Duffing equation, the equation of motion from the initial condition of $[x, \dot{x}]=[0,0]$ for the transient time of $t_{1}=1000 \mathrm{~s}$ at its first parameter and $t_{2}=10^{5} \mathrm{~s}$ for the solution time of the algorithm is solved, and the obtained bifurcation diagram is shown in Figure 25. By eliminating the non-linear term in Eq. (7), the linear frequency response of the system is shown in Figure 25(a). According to the equation of motion, the linear natural frequency of the system is $\omega=1$. For the parameters selected in Figure 25(b) and (c), the system has softening and hardening behaviors, respectively. Moreover, in Figure 25(c), the secondary resonance frequency is at $\omega_{d} \approx 0.333$. In Figure $25(\mathrm{~d})$, the validation of hardening behavior at $\omega_{d}=1.6$ is conducted, which shows very good agreement between the exact solution and the solution of the proposed algorithm in Figure 25(c).

For systems with two double-well potentials, the initial conditions or the range of frequency analysis are very important due to their considerable effect on the 


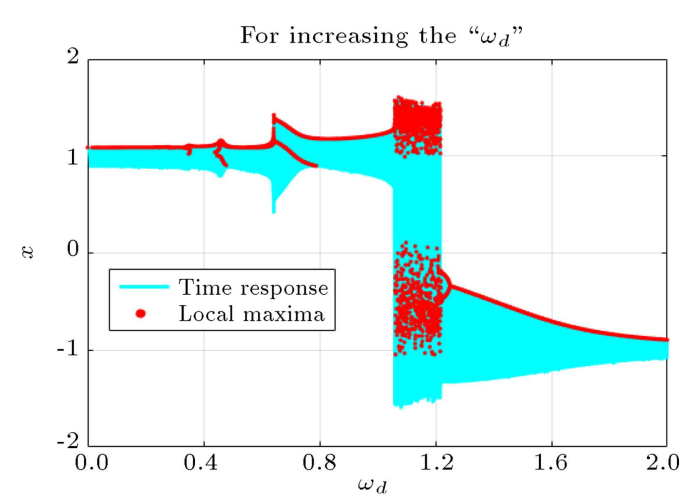

(a)

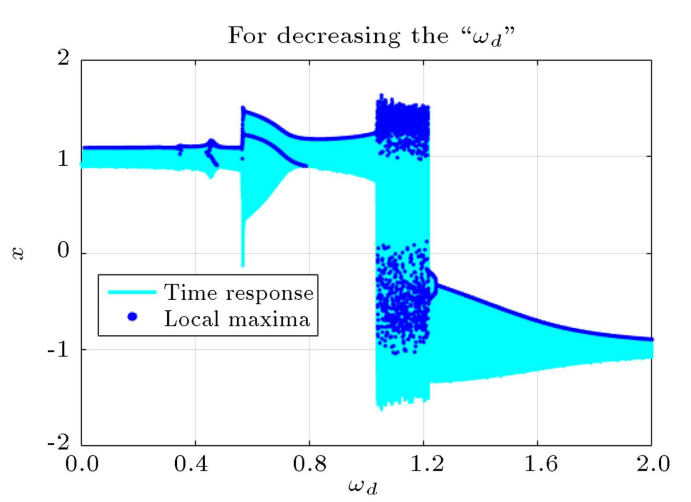

(b)

Figure 26. Frequency response of the Duffing equation with two potential wells.

system's behavior. In Eq. (7) and for $\beta<0$, the Duffing oscillator indicates the dynamism of the two potential wells. If $\alpha>0$, then two equilibrium points at $x= \pm \sqrt{-\beta / \alpha}$ are located; as a result, the system can oscillate around each of these equilibrium points, or jump from them and pass over them continuously [63].

These types of Duffing oscillators with more than one potential well are applicable to many bi-stable, tri-stable, other energy harvesters. This is due to their high performance of such systems with respect to linear systems. In fact, in these systems, power can be harvested in greater frequency ranges with higher efficiencies [64-74].

Finding a frequency range in which the system has a high-energy orbit is very important, and the analytical determination of this range for complex systems is complicated. Here, with PCM, the sensitivity of the multi-stable systems to initial conditions is analyzed, and a simple strategy to find a range of high-energy orbits and initial conditions is expressed.

The parameters of $\gamma=0.2, \delta=0.05, \alpha=1$, and $\beta=-1$ are selected. By considering these parameters, equilibrium points are located at $x= \pm 1$. By selecting zero initial conditions and starting low excitation frequency for forward at $\omega=0$ and backward solutions at $\omega=2$, the amplitude-frequency solution shown in Figure 26 is obtained. According to this time response, it is clear that the system oscillates in both wells and can swing between them.

However, this system can exhibit more hidden behaviors, which may be revealed. In Figure 27, the above simulation is repeated; however, in this case, instead of the simulation of the problem from $\omega=0$ to $\omega=2$, in Figure 27(a) and (b), the frequency range of $\omega=0$ to $\omega=1$ is selected and, then, the solution stops. As expected, these figures are similar to Figure 26. However, if the frequency interval of $\omega=1$ to $\omega=2$ is selected and the initial condition is zero, the steady state response according to Figure 27(c) and (d) is completely different; moreover, if the frequency increases or decreases again, the frequency response curve is different from the previous case. In this case, the system switches to its higher orbits. These highenergy orbits are very important in computational and physical terms. According to Figure 27(b) and (c), this range is within $0.52<\omega_{d}<1.73$.

To prove the validity of the obtained solution, Figure 28 shows the time response of the equation of motion in frequencies of $\omega_{d}=0.55$ and 1.7. The obtained result shows that the exact solution and algorithm's solutions are completely similar.

In fact, different behaviors similar to those shown in Figures 26 and 27 exist, and they can be identified through variations in the initial condition and by breaking the frequency range.

\section{Conclusion}

This study presented an algorithm in the time domain to simply obtain the bifurcation diagram and reveal the hidden behavior of the system. This algorithm basically ensures that, after the initial steady solution and at the minimum value of the parameter, if the solution time of the system increases and the gradual change of the control parameter of the system is applied, then the system is continuously in its steady state; in addition, by obtaining the peaks of the system's response, the bifurcation diagram for that the control parameter is determined.

With this concept applied, bifurcation diagrams of the chaotic systems such as Chua, Lorenz, and Rossler are analyzed. Moreover, the validation results demonstrate the high accuracy and proper functioning of the algorithm. By applying this algorithm, a hidden attractor in Chua's circuit and the Lorenz system were investigated. Then, in the same case, amplitudefrequency diagrams of the Duffing equation for the linear, hardening, and softening cases were assessed, indicating the good performance of this algorithm. Finally, the Duffing equation with two potential wells was investigated, and the sensitivity of the Duffing equation in this case was investigated by the pro- 


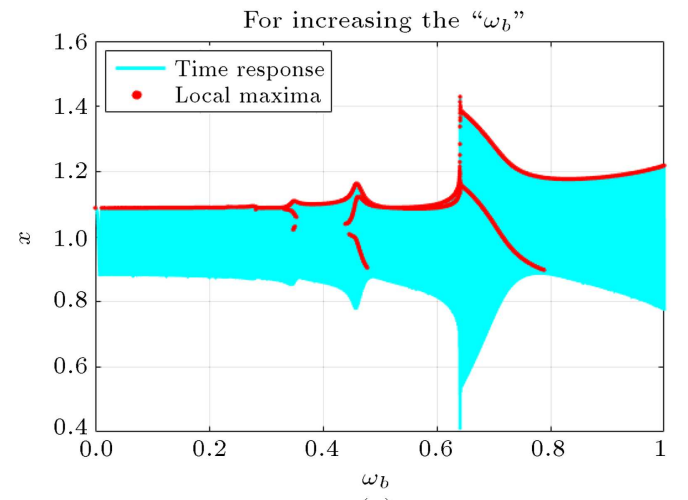

(a)

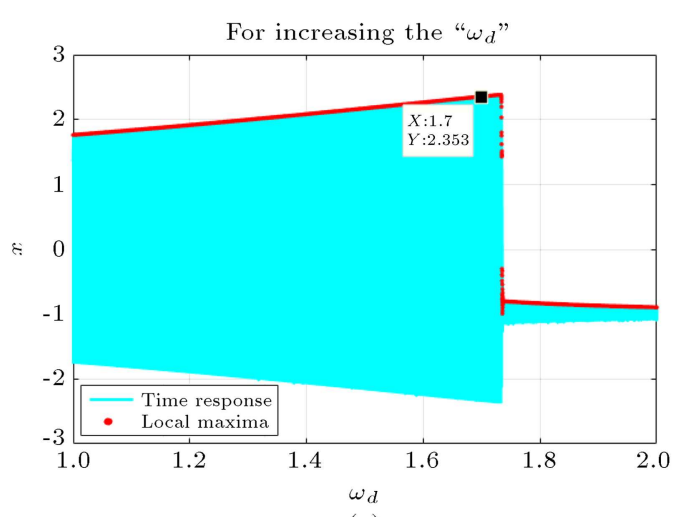

(c)

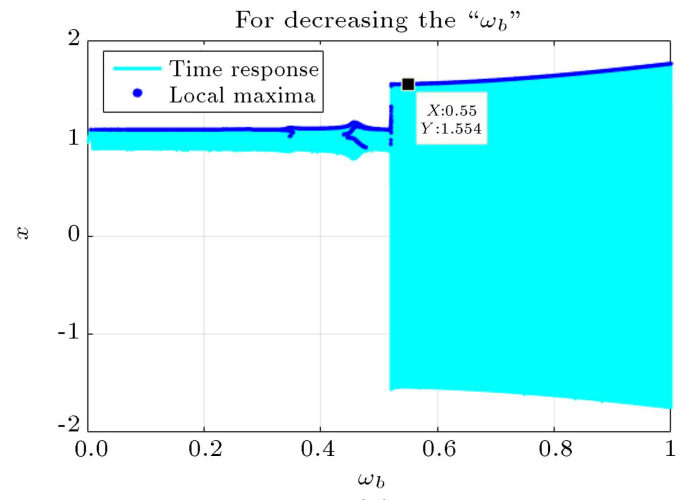

(b)

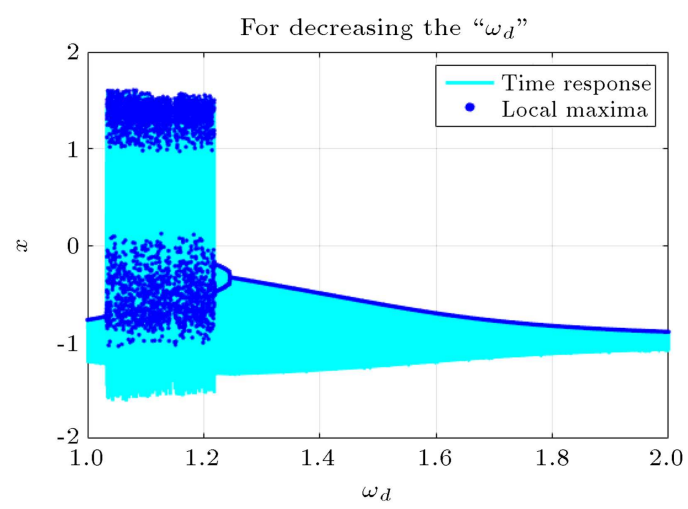

(d)

Figure 27. High and low energy orbits of the Duffing equation with two potential wells obtained by this new algorithm.

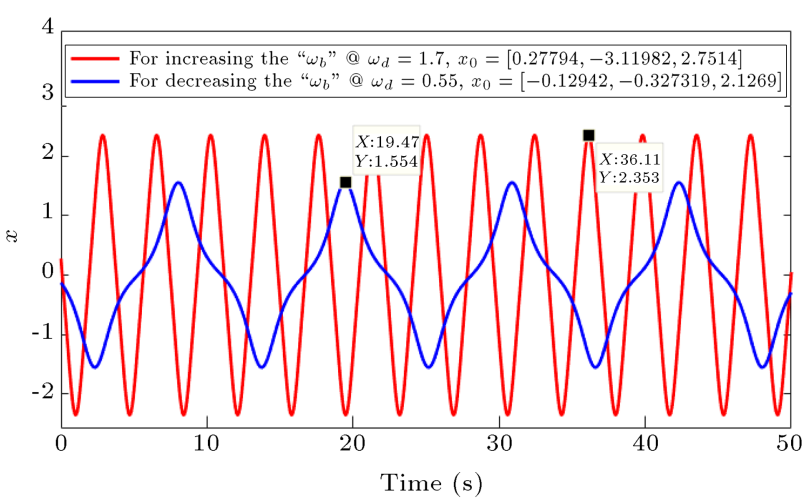

Figure 28. Validation of high energy orbits obtained by the proposed algorithm with the equation's numerical solution.

posed new approach. According to the literature, the prediction of the hidden behavior of this system with available analytical, semi-analytical, or numerical solutions was not simple. Due to the simplicity of the algorithm, this algorithm can be simply implemented by software applications that have the ability to solve ODE problems. This method can be added easily to available continuation software applications such as MANLAB, AUTO, and MATCONT.

In a typical system with a dual-core processor and $2.9 \mathrm{GH}$ frequency, the amplitude-frequency diagrams of the Duffing equation can be found in the fraction of a second, and the bifurcation diagrams of Chua's circuit, Lorenz, and Rossler systems can be obtained with very high accuracy in about one to eight minutes. The key point here is that with a negligible error and in the fraction of a second, the amplitude-frequency or bifurcation diagram of the complex systems can be obtained; in addition, after a glance view of this initially obtained bifurcation diagram, each area of diagram can be carefully investigated again. Of course, this second investigation can be achieved in a shorter amount of time.

By applying the proposed algorithm in different case studies, it is clear that this method is of high accuracy. At bifurcation points with an increase in periods of response, it is better to use backward solution. Further, at bifurcation points with a decrease in periods of solution, forward solution is preferable.

Briefly, the main advantage of the PCM method is its low computation time and the greater resolution of the bifurcation diagram; the forward and backward solutions in transition from chaos lead to the acquisition of the hidden attractor, which in fact implies the application of different initial conditions in the conventional methods. However, for example, a problem like obtaining a nonlinear frequency-amplitude response in the Duffing equation is considered; now, the question 
is: is it easy to find appropriate initial conditions to reach different oscillation regions?

In the conventional method, finding the hidden attractor is probabilistic to some extent. However, in the proposed method, based on the continuous nature of the proposed solution method, in the transition from chaos, in forward and backward solutions, the present state will determine the future state. In a nutshell, compared to the random selection of initial condition in the conventional method, the past state of the system is used to find the hidden attractor in PCM.

\section{Future works}

This algorithm is written in its simplest form with the aim of easy implementation. Further improvement can be made in this method; for example, with the nonlinear change of the control parameter, the accuracy of bifurcation points can be improved. This study did not address the type of bifurcation, existence and the location of unstable behavior, stability analysis, isolated curve, etc., which can be the subject of future works.

\section{References}

1. Nayfeh, A.H., Introduction to Perturbation Techniques, Wiley Classics Library Edition Published (1993).

2. Pakdemirli, M., Karahan, M.M.F., and Boyac, H. "A new perturbation algorithm with better convergence properties: Multiple scales lindstedt poincare method", Math. Comp. App. 14, pp. 31-44 (2009).

3. Pakdemirli, M. "Review of the new perturbationiteration method", Math. Comp. App., 18, pp. 139151 (2013).

4. Damil, N. and Potier-Ferry, M. "A new method to compute perturbed bifurcations: application to the buckling of imperfect elastic structures", Int. J. Eng. Sci., 28, pp. 943-957 (1990).

5. Vannucci, P., Cochelin, B., Damil, N., and PotierFerry, M. "An asymptotic-numerical method to compute bifurcating branches", Int. J. Num. Meth. Eng,. 41, pp. 1365-1389 (1998).

6. Boutyour, E.H., Zahrouni, H., Potier-Ferry, M., and Boudi, M. "Bifurcation points and bifurcated branches by an asymptotic numerical method and Padé approximants", Int. J. Num. Meth. Eng, 60, pp. 1987-2012 (2004).

7. Cochelin, B., Damil, N., and Potier-Ferry, M. "Asymptotic numerical method and padé approximants for nonlinear elastic structures", Int. J. Num. Meth. Eng., 37, pp. 1187-1213 (1994).

8. Hamdaoui, A., Hihi, R., Braikat, B., Tounsi, N., and Damil, N. "A new class of vector padé approximants in the asymptotic numerical method: Application in nonlinear 2D elasticity", World J. Mech., 4, pp. 44-53 (2014).
9. Elhage-Hussein, A., Potier-Ferry, M., and Damil, N. "A numerical continuation method based on Pade approximants", Int. J. Sol. Struc., 37, pp. 6981-7001 (2000).

10. Cochelin, B. "A path-following technique via an asymptotic-numerical method", Comp. Struc., 53, pp. 1181-192 (1994).

11. Kerschen, G., Peeters, M., Golinval, J.C., and Vakakis, A.F. "Nonlinear normal modes, Part I: A useful framework for the structural dynamics", Mech. Sys. Sig. Proc. 23, pp. 170-194 (2009).

12. Peeters, M., Viguié, R., Sé randour, G., Kerschen, G., and Golinval, J.C. "Nonlinear normal modes, Part II: Toward a practical computation using numerical continuation techniques", Mech. Sys. Sig. Proc., 23, pp. 195-216 (2009).

13. Barton, D.A.W., Krauskopf, B., and Wilson, R.E. "Homoclinic bifurcations in a neutral delay model of a transmission line oscillator", Nonlinearity, 20, pp. 809-829 (2007).

14. Beyn, W.J. "The numerical computation of connecting orbits in dynamical systems", IMA J. Num. Ana., 10, pp. 379-405 (1990).

15. Beyn, W.J. "Numerical methods for dynamical systems", W. Light, Ed., Advances in Numerical Analysis, Lancaster, Clarendon, Oxford, I, pp. 175-236 (1990).

16. Keller, H.B., Lectures on Numerical Methods in Bifurcation Problems, Tata Institute of Fundamental Research (1986).

17. Allgower, E.L. and Georg, K., Introduction to Numerical Continuation Methods, Springer Series in Computational Mathematics (1990).

18. Meijer, H., Dercole, F., and Oldman, B. "Numerical bifurcation analysis", Eds., R., Meyers, Mathematics of Complexity and Dynamical Systems, Springer, New York, NY (2012).

19. Doedel, E.J., Nonlinear Numerics, J., Franklin Inst., 334(5-6), pp. 1049-1073 (1997).

20. Karkar, S., Cochelin, B., and Vergez, Ch. "A highorder, purely frequency based harmonic balance formulation for continuation of periodic solutions: The case of non-polynomial nonlinearities", J. Sound Vib., 332, pp. 968-977 (2013).

21. Karkar, S., Cochelin, B., and Vergez, Ch. "A comparative study of the harmonic balance method and the orthogonal collocation method on stiff nonlinear systems", J. Sound Vib., 333, pp. 2554-2567 (2014).

22. Rega, G. and Troger, H. "Dimension reduction of dynamical systems: methods, models, applications", Nonlinear Dyn, 41, pp. 1-15 (2005).

23. Steindl, A. and Troger, H. "Methods for dimension reduction and their application in nonlinear dyn", Int. J. Sol. Struc., 38, pp. 2131-2147 (2001).

24. Terragni, F. and Vega, J.M. "On the use of POD-based ROMs to analyze bifurcations in some dissipative systems", Phys. D., 241, pp. 1393-1405 (2012). 
25. Couplet, M., Basdevant, C., and Sagaut, P. "Calibrated reduced-order POD- Galerkin system for fluid flow modeling", J. Comp. Phys., 207, pp. 192-220 (2005).

26. Sirisup, S., Karniadakis, G.E., and Kevrekidis, I.G. "Equations-free/Galerkin-free POD assisted computation of incompressible flows", J. Comp. Phys., 207, pp. $568-587$ (2005).

27. Rapun, M.L. and Vega, J.M. "Reduced order models based on local POD plus Galerkin projection", $J$. Comp. Phys., 229, pp. 3046-3063 (2010).

28. Terragni, F., Valero, E., and Vega, J.M. "Local POD plus Galerkin projection in the unsteady lid-driven cavity problem", J. Sci. Comp. arch., 33, pp. 35383561 (2011).

29. Doedel Laurette, E., Tuckerman, S., Numerical Methods for Bifurcation Problems and Large-Scale Dynamical Systems, Springer, The IMA Volumes in Mathematics and Its Applications, 119 (1999).

30. Govaerts, W. "Numerical bifurcation analysis for ODEs", J. Comp. App. Math., 125, pp. 57-68 (2000).

31. Heyman, J., Girault, G., Guevel, Y., Allery, C., Hamdouni, A., and Cadou, J.M. "Computation of Hopf bifurcations coupling reduced order models and the asymptotic numerical method", Comp. Fluids, 76, pp. $73-85$ (2013).

32. Meyers, R.A., Mathematics of Complexity and Dynamical Systems, Springer Science + Business Media, LLC (2012).

33. Dijkstra, H.A., Wubs, F.W., Cliffe, A.K., et al. "Numerical bifurcation methods and their application to fluid dynamics: Analysis beyond simulation", Comm. Comp. Phy., 15, pp. 1-45 (2014).

34. Gai, G. and Timme, S. "Nonlinear reduced-order modelling for limit-cycle oscillation analysis", Nonlinear Dyn, 84, pp. 991-1009 (2016).

35. Feng, Y. and Pan, W. "Hidden attractors without equilibrium and adaptive reduced-order function projective synchronization from hyper chaotic Rikitake system", Pramana J. Phy., 88, p. 62 (2017).

36. Chnafa, C., Valen-Sendstad, K., Brina, O., Pereira, V.M., and Steinman, D.A. "Improved reduced-order modelling of cerebrovascular flow distribution by accounting for arterial bifurcation pressure drops", $J$. Biom, 51, pp. 83-88 (2017).

37. Amabili, M., Karazis, K., and Khorshidi, K. "Nonlinear vibrations of rectangular laminated composite plates with different boundary conditions", Int. $J$. Struc. Stab. Dyn., 11, pp. 673-695 (2011).

38. Kurt, E., Ciylan, B., Taskan, O.O., and Kurt. H.H. "Bifurcation analysis of a resistor-double inductor and double diode circuit and a comparison with a resistorinductor-diode circuit in phase space and parametrical responses", Scientia Iranica, Trans. D: Computer Sci. Eng. Ele. 21, pp. 935-944 (2014).
39. Sayyaadi, H., Tadayon, M.A., and Eftekharian, A.A. "Micro resonator nonlinear dynamics considering intrinsic properties", Scientia Iranica, Trans. B: Mech. Eng., 16, pp. 121-129 (2009).

40. http://manlab.lma.cnrs-mrs.fr/

41. https://sourceforge.net/p/cocotools/wiki/Home/

42. http://www.ni.gsu.edu/ rclewley/PyDSTool/ FrontPage.html

43. http://indy.cs.concordia.ca/auto/\#documentation

44. Lorenz, E.N. "Deterministic nonperiodic flow", J. Atm. Sci., 20, pp. 130-141 (1963).

45. Rossler, O.E. "An equation for continuous Chaos", Physics Letters, 57A, pp. 397-398 (1976).

46. Chua, L.O. and Lin, G.N. "Canonical realization of Chua's circuit family", IEEE Tran. Circuits Sys., 37, pp. 885-902 (1990).

47. Chen, G. and Ueta, T. "Yet another chaotic attractor", Int. J. Bifu. Chaos, 9, pp. 1465-1466 (1999).

48. Dudkowski, D., Jafari, S., Kapitaniak, T., Kuznetsov, N.V., Leonov, G.A., and Prasad, A. "Hidden attractors in dynamical systems", Phy. Rep., 637, pp. 1-50 (2016).

49. Kuznetsov, N.V., Leonov, G.A., and Vagaitsev, V.I. "Analytical-numerical method for attractor localization of generalized Chua's system", IFAC Proc., 43, pp. 29-33 (2010).

50. Leonov, G.A., Vagaitsev, V.I., and Kuznetsov, N.V. "Algorithm for localizing Chua attractors based on the harmonic linearization method", Doklady Math., 433, pp. 323-326 (2010).

51. Leonov, G.A., Kuznetsov, N.V., and Vagaitsev, V.I. "Localization of hidden Chua's attractors", Phy. Let. A, 375, pp. 2230-2233 (2011).

52. Leonov, G.A. and Kuznetsov, N.V. "Algorithms for searching for hidden oscillations in the Aizerman and Kalman problems", Doklady Math., 84(1), 475-481 (2011).

53. Leonov, G.A. and Kuznetsov, N.V. "Analyticalnumerical methods for investigation of hidden oscillations in nonlinear control systems", IFAC Proc, 44(1), pp. 2494-2505 (2011).

54. Bragin, V.O., Vagaitsev, V.I., Kuznetsov, N.V., and Leonov, G.A. "Algorithms for finding hidden oscillations in nonlinear systems. The Aizerman and Kalman Conjectures and Chua's circuits", J. Computer Sys. Sci. Int., 50(4), pp. 511-543 (2011).

55. Kuznetsov, N., Kuznetsova, O., Leonov, G., and Vagaitsev, V. "Analytical-numerical localization of hidden attractor in electrical Chua's circuit", Informatics in Control, Automation and Robotics. Lecture Notes in Electrical Engineering, 174, Springer, Berlin, Heidelberg, pp. 149-157 (2013).

56. Zelinka, I. "Evolutionary identification of hidden chaotic attractors", Eng. App. Art. Intel., 50, pp. 159167 (2016). 
57. Ivancevic, V.G., Ivancevic, T.T., High-Dimensional Chaotic and Attractor Systems: A Comprehensive Introduction, Intelligent Systems, Control and Automation: Science and Engineering, 1st edition, Springer (2007).

58. Li, Q., Zeng, H., and Yang, X.S. "On hidden twin attractors and bifurcation in the Chua's circuit", Nonlinear Dyn, 77(1-2), pp. 255-266 (2014).

59. Griffiths, D.F. and Higham, D.J. Numerical Methods for Ordinary Differential Equations: Initial Value Problems, Springer-Verlag London (2010).

60. Dormand, J.R. and Prince, P.J. "A family of embedded Runge-Kutta formula", J. Comp. App. Math., 6, pp. 19-26 (1980).

61. http://www.scholarpedia.org/article/Rossler attractor.

62. Kovacic, I. and Brennan, M.J., The Duffing Equation: Nonlinear Oscillators and Their Behaviour, Wiley (2011).

63. http://www.scholarpedia.org/article/Duffing oscillator.

64. Harne, R.L. and Wang, K.W. "A review of the recent research on vibration energy harvesting via bistable systems", Smart Mat. Struc., 22(2), 023001 (2013).

65. Panyam, M. and Ram, M., "Characterizing the effective bandwidth of nonlinear vibratory energy harvesters possessing multiple stable equilibria", $\mathrm{PhD}$ Dissertation, Clemson University, December (2015).

66. Ramlan, R., Brennan, M.J., Mace, B.R., and Kovacic, I. "Potential benefits of a non-linear stiffness in an energy harvesting device", Nonlinear Dyn, 59(4), pp. $545-558$ (2010).

67. Erturk, A. and Inman, D.J. "Broadband piezoelectric power generation on high-energy orbits of the bistable Duffing oscillator with electromechanical coupling", $J$. Sound Vib., 330, pp. 2339-2353 (2011).

68. Karami, M.A. and Inman, D.J. "Equivalent damping and frequency change for linear and nonlinear hybrid vibrational energy harvesting systems", J. Sound Vib., 330, pp. 5583-5597 (2011).
69. Panyamn, M., Masana, R., and Daqaq, M.F. "On approximating the effective bandwidth of bi-stable energy harvesters", Int. J. Nonl. Mech., 67, pp. 153163 (2014).

70. Panyam, M. and Daqaq, M.F. "A comparative performance analysis of electrically optimized nonlinear energy harvesters", J. Intel. Mate. Struc., 27(4), pp. 537-548 (2016).

71. Guang-Qing, W. and Wei-Hsin, L. "A strategy for magnifying vibration in high-energy orbits of a bistable oscillator at low excitation levels", Chinese Phy. Let., 32(6), 068503 (2015).

72. Zhou, Sh., Cao, J., Inman, D.J., Liu, Sh., Wang, W., and Lin, J. "Impact-induced high-energy orbits of nonlinear energy harvesters", Ap. Phy. Let., 106, 093901 (2015).

73. Wang, G.Q. and Liao, W.H. "A bistable piezoelectric oscillator with an elastic magnifier for energy harvesting enhancement", J. Intel. Mate. Struc., 28(3), pp. 392-407 (2017).

74. Zhou, Sh., Cao, J., Inman, D.J., Lin, J., and Li, D. "Harmonic balance analysis of nonlinear tristable energy harvesters for performance enhancement", $J$. Sound Vib., 373(7), pp. 223-235 (2016).

\section{Biographies}

Hossein Asghari received BS and MS degrees in Mechanical Engineering from Urmia University of Technology and Babol Noshirvani University of Technology, Iran in 2015 and 2018, respectively. His research interests include nonlinear dynamic and vibration, energy harvesting, smart materials, and fluid-structure interaction.

Morteza Dardel received a $\mathrm{PhD}$ degree from Amirkabir University, Tehran, Iran in Solid Mechanics in 2009 and is currently an Assistant Professor in the Faculty of Mechanical Engineering at Babol Noshirvani University of Technology, Iran. His research interests are aeroelasticity, nonlinear dynamic, vibration, control of continuous systems, and smart structures. 